Freiburg THEP-93/27

gr-qc/9312015

\title{
THE SEMICLASSICAL APPROXIMATION TO QUANTUM GRAVITY
}

\author{
Claus Kiefer \\ Fakultät für Physik, Universität Freiburg, Hermann-Herder-Str. 3, \\ D-79104 Freiburg, Germany
}

\begin{abstract}
A detailed review is given of the semiclassical approximation to quantum gravity in the canonical framework. This includes in particular the derivation of the functional Schrödinger equation and a discussion of semiclassical time as well as the derivation of quantum gravitational correction terms to the Schrödinger equation. These terms are used to calculate energy shifts for fields in De Sitter space and non-unitary contributions in black hole evaporation. Emphasis is also put on the relevance of decoherence and correlations in semiclassical gravity. The back reaction of non-gravitational quantum fields onto the semiclassical background and the emergence of a Berry connection on superspace is also discussed in this framework.
\end{abstract}

December 1993

\footnotetext{
${ }^{1}$ To appear in Canonical gravity - from classical to quantum, edited by J. Ehlers and H. Friedrich (Springer, Berlin, 1994).
} 


\section{Chapter 1}

\section{Introduction}

Despite many efforts in the last sixty years there does not yet exist a consistent theory of quantum gravity. Why, then, should one address an issue like the semiclassical approximation to quantum gravity if there is no theory available which one could approximate? The answer is simple. According to the correspondence principle, the notion of a classical spacetime should emerge in an appropriate limit from quantum theory. Thus, by applying semiclassical considerations one hopes to get an insight into some of the structures of the full, elusive, theory.

Semiclassical ideas have been very important in the history of quantum theory. The Bohr-Sommerfeld quantization formula

$$
\frac{1}{2 \pi \hbar} \oint p d x=n+\frac{1}{2},
$$

for example, was used before the advent of quantum mechanics as an ad hoc assumption to explain atomic spectra. Formula (1.1) can, of course, be recovered from quantum mechanics by applying WKB methods to the Schrödinger equation and is even equal to the exact result in some special cases such as the harmonic oscillator or the Coulomb potential. Higher order terms in a WKB expansion then yield corrections to (1.1) of the order of $\lambda / L$, where $\lambda$ is the de Broglie wavelength, and $L$ is the typical dimension of the system under consideration.

A more impressive application of semiclassical ideas is Schrödinger's approach to his wave equation by using Hamiltonian methods. These methods had been developed in the 19th century to provide a joint formalism of optics and mechanics, which was then, however, merely regarded to give a formal analogy without physical significance, since there was no obvious relation between the velocity of light and the velocity of a mechanical particle. This changed only with de Broglie who came up with the idea that particles are waves. It then seemed natural for Schrödinger to look for a wave equation which yields the Hamilton-Jacobi equation in some appropriate limit. What 
he basically did in his pioneering work (Schrödinger, 1926a) was to take the Hamilton-Jacobi equation円

$$
H\left(q_{i}, \frac{\partial S}{\partial q^{i}}\right) \equiv \frac{1}{2 m}(\nabla S)^{2}+V=E
$$

and make the following ansatz for a wave function:

$$
\psi \equiv \exp (i S / \hbar)
$$

(The constant $\hbar$ was first left open and later determined from the spectrum of the hydrogen atom.) Calculating the second derivatives of $\psi$ with respect to the $q_{i}$ and neglecting the second derivatives of $S$ compared to its first derivatives (this is motivated by geometrical optics where $S$ varies very little with $x$ ) he found after insertion into (1.2) the famous Schrödinger equation

$$
\left(-\frac{\hbar^{2}}{2 m} \nabla^{2}+V\right) \psi=E \psi .
$$

In contrast to de Broglie, Schrödinger has only waves, but they are waves in configuration space and not in three-dimensional space like light waves. Semiclassical considerations of this kind will also be the general theme in this article, but instead of starting from the Hamilton-Jacobi equation we will accept a "model equation" for the wave functionals in quantum gravity and then discuss consequences from the semiclassical expansion of this equation.

To present a last example of the importance of semiclassical considerations in the history of quantum theory, I would like to write down the corrections to the classical Maxwell action through quantum fluctuations of electrons and positrons, which was found by Heisenberg and Euler (1936) before the development of QED. In the weak field approximation the corrected Maxwell Lagrangian reads

$$
\mathcal{L}=\frac{1}{8 \pi}\left(\mathbf{E}^{2}-\mathbf{B}^{2}\right)+\frac{e^{4} \hbar}{360 \pi^{2} m^{4} c^{7}}\left[\left(\mathbf{E}^{2}-\mathbf{B}^{2}\right)^{2}+7(\mathbf{E B})^{2}\right] .
$$

This result can of course be found from full QED by "integrating out" the fermions and expanding in powers of $\hbar$. Since this is equivalent to an expansion in the number of loops, (1.5) is also called the one-loop effective Lagrangian. We will encounter an analogous level of approximation in the case of quantum gravity below. The knowledge of (1.5) allows the computation of physical processes such as the scattering of light by external fields (Delbrück scattering), which has been experimentally observed.

\footnotetext{
${ }^{1}$ In this paper he treats the stationary case. The time-dependent Schrödinger equation was introduced only in his last paper on quantization as an eigenvalue problem (Schrödinger, 1926b).
} 
Semiclassical methods have also become increasingly popular in the field of chaos where people have even coined the term "postmodern quantum mechanics" for these methods.

What is the situation in quantum gravity? It would be ideal to start with a "theory of everything" like superstring theory which encompasses all interactions including gravity in a single quantum framework. Unfortunately, string theory has not yet reached the stage where any genuine quantum gravity predictions such as black hole evaporation can be made. A great deal of work has therefore been done on the level of quantum general relativity, the topic of this volume, where formal quantization rules are applied to general relativity without invoking any scheme of unification of interactions. Whether quantum general relativity exists as a consistent theory and whether it can be derived from an underlying "theory of everything" in an appropriate low-energy limit is an open issue (it depends in particular on the ratio of, say, the string scale to the Planck scale). As long as the status of a possible fundamental theory is unknown, it seems perfectly justified to investigate whether quantum general relativity can serve as a consistent theory of quantum gravity. The perturbative non-renormalisability of this approach may thus not necessarily present a fundamental problem. (In the following we will always use the term "quantum gravity" in this sense.) While most of the other contributions in this volume are concerned with the fundamental level itself, this article will basically address the following questions. Firstly, how can the framework of quantum field theory in a given background spacetime be recovered, at least formally, from quantum gravity and secondly, can one calculate corrections to this framework which come from quantum gravity and may even be testable? Within the framework of quantum field theory in a given spacetime one can derive concrete results like the Hawking temperature for black holes. It would thus be interesting to know the corrections to such results from quantum gravity.

The organisation of this article is as follows. In the next section I show how the functional Schrödinger equation for matter fields can formally be recovered from quantum gravity. I also present the nonrelativistic limit of the Klein-Gordon equation as a useful formal analogy.

Section 3 is a major part of this contribution and is devoted to the derivation of correction terms to the Schrödinger equation from quantum gravity. Applications include the corrections to energy expectation values of scalar fields in de Sitter space and non-unitary contributions to the evaporation of black holes. I also make a comparison with an analogous approximation scheme in QED.

In section $4 \mathrm{I}$ address the issues of decoherence and back reaction, which are important concepts in the semiclassical expansion. I briefly discuss examples from quantum mechanics for illustration and then turn to the analogous situation in quantum gravity. The modification of semiclassical time through 
back reaction is carefully examined.

The last section gives a brief summary and a brief account of the topics which have not been addressed in this article. This includes the relevance of semiclassical considerations for the problem of time in quantum gravity as well as a comparison with the effective action approach and standard perturbation theory. 


\section{Chapter 2}

\section{Derivation of the Schrödinger equation from quantum gravity}

The central equation of canonical quantum gravity is the constraint equation $H \Psi=0$. This was discussed in detail in other contributions to this volume. In the following we will mainly use the geometrodynamical language, since semiclassical discussions are most transparent in this picture. We will comment, however, on the situation using Ashtekar's variables at the end of this section.

Starting point is the full Wheeler-DeWitt equation

$\mathcal{H} \Psi\left[h_{a b}, \phi\right] \equiv\left(-\frac{16 \pi G \hbar^{2}}{c^{2}} G_{a b c d} \frac{\delta^{2}}{\delta h_{a b} \delta h_{c d}}-\frac{c^{4}}{16 \pi G} \sqrt{h}(R-2 \Lambda)+\mathcal{H}_{m}\right) \Psi=0$

where $h_{a b}$ is the three-metric, $R$ the three-dimensional Ricci scalar, $\Lambda$ the cosmological constant, and $\mathcal{H}_{m}$ is the Hamiltonian density for nongravitational fields, denoted symbolically below by $\phi$. In the remaining part of this article we will deal for simplicity exclusively with scalar fields. There is of course the usual factor ordering ambiguity in (2.1), but for definiteness we use the naive factor ordering with no first metric derivatives in the following discussion and comment on the general case briefly at the end.

One now introduces the parameter

$$
M \equiv \frac{c^{2}}{32 \pi G}
$$

with respect to which the semiclassical expansion will be performed. The Wheeler-DeWitt equation then reads

$$
\left(-\frac{\hbar^{2}}{2 M} G_{a b} \frac{\delta^{2}}{\delta h_{a} \delta h_{b}}+M V+\mathcal{H}_{m}\right) \Psi=0
$$


where $V$ stands for $-2 c^{2} \sqrt{h}(R-2 \Lambda)$, and we have introduced a condensed notation, labeling three-metric coefficients by $h_{a}$ and components of the DeWitt-metric by $G_{a b}$, since contractions always involve index pairs.

An expansion with respect to the large parameter $M$ should lead to sensible results if the relvant mass scales of non-gravitational fields are much smaller than the Planck mass. If there were no non-gravitational fields, an $M$ expansion would be fully equivalent to an $\hbar$ expansion and thus to the usual WKB expansion for the gravitational field (this can be seen from (2.1) after multiplication with $G$ ). In the presence of non-gravitational fields, the $M$ expansion is analogous to a Born-Oppenheimer expansion, where the large mass of the nuclei is replaced by $M$, and the small electron mass is replaced by the mass-scale of the non-gravitational field.

The first subsection is thus devoted to a brief review of the Born-Oppenheimer approximation in molecular physics. Most of the steps in that scheme will simply be extrapolated, in a formal way, to quantum gravity. We then present another helpful analogy - the nonrelativistic expansion of the Klein-Gordon equation. Subsection 3 will then present a detailed discussion of the derivation of the Schrödinger equation from the Wheeler-DeWitt equation, while the last section will give a brief review of the analogous situation in the Ashtekar framework.

\subsection{Born-Oppenheimer approximation}

Consider the following quantum mechanical Hamiltonian

$$
H=\frac{P^{2}}{2 M}+\frac{p^{2}}{2 m}+V(R, r) \equiv \frac{P^{2}}{2 M}+h,
$$

where $M \gg m$. We seek an approximate solution to the stationary Schrödinger equation

$$
H \Psi=E \Psi .
$$

We assume that the spectrum of the "light" particle is known for each configuration $R$ of the "heavy" particle, i.e.

$$
h|n ; R\rangle=\epsilon(R)|n ; R\rangle .
$$

The full state $\Psi$ is then expanded into these eigenfunctions,

$$
\Psi=\sum_{k} \psi_{k}(R)|k ; R\rangle .
$$

If one inserts this ansatz into (2.5) one finds, after multiplication with $\langle n ; R|$ from the left and using the fact that these states are orthonormalised, an 
equation for the wave functions $\psi_{n}$,

$$
\begin{aligned}
& \left(-\frac{\hbar^{2}}{2 M} \nabla_{R}^{2}+\epsilon_{n}(R)-E\right) \psi_{n}(R)= \\
& \frac{\hbar^{2}}{M} \sum_{k}\left\langle n ; R \mid \nabla_{R} k ; R\right\rangle \nabla_{R} \psi_{k}(R)+\frac{\hbar^{2}}{2 M} \sum_{k}\left\langle n ; R \mid \nabla_{R}^{2} k ; R\right\rangle \psi_{k}(R)
\end{aligned}
$$

Note that this is still an exact formula. The Born-Oppenheimer approximation now consists in the neglection of off-diagonal terms in (2.7).The result is an autonomous equation for the $\psi_{n}$, which is conveniently written as follows

$$
\begin{aligned}
& \left(\frac{1}{2 M}(-i \hbar \nabla-\hbar A(R))^{2}-\frac{\hbar^{2} A^{2}}{2 M}\right. \\
& \left.-\frac{\hbar^{2}}{2 M}\left\langle n ; R \mid \nabla_{R}^{2} n ; R\right\rangle+\epsilon_{n}(R)\right) \psi_{n}(R)=E \psi_{n}(R),
\end{aligned}
$$

where we have introduced the "Berry connection"

$$
A(R)=-i\left\langle n ; R \mid \nabla_{R} n ; R\right\rangle
$$

Note that the momentum of the "slow" particle has been shifted according to

$$
P \rightarrow P-\hbar A
$$

In most textbook treatments this connection is not taken into account, since it is assumed that the "fast" eigenfunctions can be chosen to be real, in which case the connection $A$ vanishes. There are, however, situations where this cannot be done and $A$ has to be taken into account, see for example Jackiw (1988a), and Wudka (1990). As we will see below, the complex nature of the "fast" wave functions is essential in quantum gravity.

We emphasise that the essential approximation in this scheme consists in the neglection of the off-diagonal terms in (2.7) and thus the reduction of the superposition (2.6) to a single product state. As I will briefly review in section 4 below, this can be justified by taking the unavoidable interaction with environmental degrees of freedom into account - the various components in (2.6) decohere from each other. Moreover, this interaction leads to decoherence within one component. The position of heavy molecules, for example, is "measured" by radiation (Joos and Zeh, 1985). In this sense one can therefore justify the substitution of a fixed position $R_{0}$ of the "heavy" particle into the "fast" states $\langle n ; R|$. It is only in this limit that molecules with a well-defined shape emerge. 


\subsection{Non-relativistic limit of the Klein-Gordon equation}

The Klein-Gordon equation reads, in the case of vanishing external field,

$$
\left(\frac{\hbar^{2}}{c^{2}} \frac{\partial^{2}}{\partial t^{2}}-\hbar^{2} \nabla^{2}+m^{2} c^{2}\right) \varphi(\mathbf{x}, t)=0 .
$$

On comparison with (2.3) we recognise that the limit $M \rightarrow \infty$ is equivalent to the limit $c \rightarrow \infty$ in the Klein-Gordon case. It is, however, emphasised that this analogy is only a partial one. The Klein-Gordon equation (2.11) is an equation for a one-particle wave function, whereas the Wheeler-DeWitt equation (2.3) is already a "second-quantized" equation, i.e. the corresponding state is a wave functional on a configuration space, whose points are field configurations. Nevertheless, both equations have the structure of a wave equation and exhibit many formal similarities.

The non-relativistic expansion starts by writing the wave function $\varphi(\mathbf{x}, t)$ as

$$
\varphi(\mathbf{x}, t)=\exp (i S(\mathbf{x}, t) / \hbar)
$$

and expanding

$$
S=c^{2} S_{0}+S_{1}+c^{-2} S_{2}+\ldots
$$

Inserting this into (2.11) and comparing equal powers of the expansion parameter $c^{2}$ yields at order $c^{4}$

$$
\left(\nabla S_{0}\right)^{2}=0
$$

so that $S_{0}$ depends on $t$ only. The next order, $c^{2}$, yields

$$
-\left(\frac{\partial S_{0}}{\partial t}\right)^{2}+m^{2}=0
$$

This is a Hamilton-Jacobi type of equation, which gives real solutions if $m^{2} \geq 0$, i.e. if there are no tachyons. The solutions can then immediately be written down:

$$
S_{0}= \pm m t+\text { constant }
$$

At this order of approximation we thus have for the wave function

$$
\varphi_{\mp}(\mathbf{x}, t) \approx \exp \left( \pm i m c^{2} t / \hbar\right) .
$$

Its interpretation is obvious: It describes a particle at rest with positive energy (lower sign) or with negative energy (upper sign). To neglect superpositions like $\varphi_{+}+\varphi_{-}$reflects the fact that field theoretic effects like pair 
creation are assumed to be negligible. Note that both $\varphi_{+}$and $\varphi_{-}$are complex, despite the real character of the Klein-Gordon equation (2.11). We assume in the following the use of the positive energy wave function.

The next order $\left(c^{0}\right)$ yields an equation for $S_{1}$ :

$$
2 m \dot{S}_{1}+\left(\nabla S_{1}\right)^{2}-i \hbar \nabla^{2} S_{1}=0 .
$$

This can be simplified by defining the wave function

$$
\chi \equiv \exp \left(i S_{1} / \hbar\right)
$$

which leads, for non-vanishing mass, to

$$
i \hbar \dot{\chi}=-\frac{\hbar^{2}}{2 m} \nabla^{2} \chi
$$

At this order one has thus recovered the non-relativistic Schrödinger equation. If one proceeds beyond this order, one finds, at order $c^{-2}$, the first relativistic corrections. The equation involving $S_{2}$ reads

$$
i \hbar \ddot{S}_{1}-i \hbar \nabla^{2} S_{2}-\dot{S}_{1}^{2}+2 m \dot{S}_{2}+2 \nabla S_{1} \nabla S_{2}=0
$$

which can be simplified if one rewrites $S_{1}$ in terms of $\chi$ and introduces the definition

$$
\psi \equiv \chi \exp \left(i S_{2} / \hbar c^{2}\right)
$$

The wave function $\psi$ then obeys the modified Schrödinger equation

$$
i \hbar \dot{\psi}=-\frac{\hbar^{2}}{2 m} \nabla^{2} \psi-\frac{\hbar^{4}}{8 m^{3} c^{2}} \nabla^{2} \nabla^{2} \psi .
$$

The last term on the right-hand side of this equation is, of course, just the first relativistic correction to the kinetic energy, which can easily be obtained by expanding the square root $\sqrt{m^{2} c^{4}+p^{2} c^{2}}$. The present expansion scheme has, however, the merit of systematically producing correction terms also in the presence of external fields. In the case of coupling to an external gravitational field, some of these correction terms are imaginary and thus explicitly violate unitarity in the matter sector (Lmmerzahl, 1993). We will encounter similar terms in the case of the Wheeler-DeWitt equation, see section 3 .

I emphasise that (2.23) does not make sense physically as a differential equation (the effective Hamiltonian, for example, is unbounded from below). It only makes sense if the additional term is used to calculate small corrections to results found on the previous level of approximation. One can, for example, 
calculate the relativistic corrections to the energy eigenvalues of hydrogenlike pionic atoms:

$$
\begin{aligned}
\Delta E_{r e l} & =-\int d^{3} x \psi_{n l m}^{*}\left(\frac{\hbar^{4}}{8 m^{3} c^{2}} \nabla^{2} \nabla^{2}\right) \psi_{n l m} \\
& =-\frac{m c^{2}}{2}(Z \alpha)^{4}\left(\frac{1}{n^{3}\left(l+\frac{1}{2}\right)}-\frac{3}{4 n^{4}}\right) .
\end{aligned}
$$

We now turn to the interesting case of the Wheeler-DeWitt equation.

\subsection{Derivation of the functional Schrödinger equation from the Wheeler-DeWitt equa- tion}

We now make an expansion of the Wheeler-DeWitt equation (2.3) with respect to $M$, which is formally similar to the non-relativistic expansion of the Klein-Gordon equation presented above. To be specific, we take $\mathcal{H}_{m}$ to be the Hamiltonian density of a minimally coupled scalar field, i.e.,

$$
\mathcal{H}_{m}=\frac{1}{2}\left(-\frac{\hbar^{2}}{\sqrt{h}} \frac{\delta^{2}}{\delta \phi^{2}}+\sqrt{h} h^{a b} \phi_{, a} \phi_{, b}+\sqrt{h}\left(m^{2} \phi^{2}+U(\phi)\right)\right),
$$

where $U(\phi)$ can be an arbitrary potential. In analogy to (2.12) we make the ansatz

$$
\Psi \equiv \exp (i S / \hbar)
$$

and expand

$$
S=M S_{0}+S_{1}+M^{-1} S_{2}+\ldots
$$

As remarked above, this expansion scheme will distinguish all gravitational degrees of freedom (as being "semiclassical") from the remaining ones (which

are "fully quantum"). One would, of course, expect that not all degrees of the gravitational field are semiclassical, such as the "gravitons." It is straightforward to generalise this scheme to incorporate the more general situation. Vilenkin (1989), for example, makes the ansatz

$$
h_{a b}=h_{a b}^{(0)}+\sqrt{8 \pi G} q_{a b} .
$$

Inserting this into the Wheeler-DeWitt equation, one recognises that the $q_{a b}$ appear on the same footing as $\phi$ and are therefore "fully quantum." The expansion is thus, of course, no longer an expansion with respect to $G$. Generally speaking, the assumption behind this is that the quantum states of the "fast variables" depend on the "semiclassical variables" only adiabatically, see e. g. Halliwell and Hawking (1985) or Kiefer (1987). 
In complete analogy to (2.14)ff. one finds after inserting (2.26) and (2.27) into (2.3) at the highest order, $M^{2}$,

$$
\left(\frac{\delta S_{0}}{\delta \phi}\right)^{2}=0,
$$

i.e. that $S_{0}$ depends only on the three-metric. If many matter fields are present one can draw this conclusion only if their kinetic energies are positive (provided, of course, $S_{0}$ is assumed to be either real or pure imaginary). Since the gravitational kinetic energy is indefinite, one is thus not able to derive the opposite limit, where all gravitational degrees of freedom are quantum and the non-gravitational ones semiclassical.

The next order, $M^{1}$, yields

$$
\frac{1}{2} G_{a b} \frac{\delta S_{0}}{\delta h_{a}} \frac{\delta S_{0}}{\delta h_{b}}+V\left(h_{a}\right)=0 .
$$

This is the Hamilton-Jacobi equation for the gravitational field (Peres, 1962). It is equivalent to all ten of Einstein's field equations (DeWitt, 1967; Gerlach, 1969; DeWitt, 1970). I want to briefly review how this equivalence can be shown, i. e. how the "dynamical laws follow from the laws of the instant" (Kuchař, 1992).

Suppose one is given a solution $S_{0}$ to $(2.29)$. From this one can immediately read off the geometrodynamical field momentum

$$
\pi^{a b}=M \frac{\delta S_{0}}{\delta h_{a b}}
$$

One half of Hamilton's equations of motion then give the connection between the field momenta and the "velocities" $\dot{h_{a b}}$,

$$
\dot{h_{a b}}=-2 N K_{a b}+N_{a \mid b}+N_{b \mid a}
$$

where $K_{a b}$ are the components of the extrinsic curvature of three-space, whose relation to the momenta is

$$
K_{a b}=-\frac{16 \pi G}{c^{2}} G_{a b c d} \pi^{c d}
$$

and $N$ and $N_{a}$ are respectively the lapse function and the shift vector, for both of which a choice must be made in order for $\dot{h_{a b}}$ to be specified. After this has been done, and an "initial" three-geometry has been chosen, (2.31) can be integrated to yield a whole spacetime with a definite foliation and a definite choice of coordinates on each member of this foliation. One can thus combine all the trajectories in superspace which describe the same spacetime to a "sheaf" (DeWitt, 1970). Lapse and shift can be chosen in such a way 
that these curves comprise a "sheaf of geodesics." Superposing many WKB solutions of the gravitational field, Gerlach (1969) has demonstrated how one specific trajectory in configuration space, i. e. one specific spacetime, can be traced out by a wave packet.

Since $S_{0}$ satisfies, in addition to the Hamilton-Jacobi equation (2.29), the three equations

$$
\left(\frac{\delta S_{0}}{\delta h_{a b}}\right)_{\mid b}=0,
$$

the "complete solution" depends on two arbitrary functions on three-space. The relations (2.33) follow from an expansion of the momentum constraints in powers of $M$. As was shown by Moncrief and Teitelboim (1972), these equations follow from the validity of the Hamilton-Jacobi equation (2.29) itself, if one takes into account the Poisson bracket relations between the constraints $\mathcal{H}$ and $\mathcal{H}_{a}$. While (2.29) and (2.33) are equivalent to the $G_{00}$ and $G_{0 i}$ part of the Einstein equations, the remaining six field equations can be found by differentiating (2.31) with respect to $t$ and eliminating $S_{0}$ by making use of (2.31) and (2.29). Basically, this is the same procedure as can already be done in Newtonian mechanics.

The Hamilton-Jacobi equation (2.29), as it stands, is actually an infinity of equations, one at each space point. An alternative viewpoint is also useful (Giulini, 1993). It interprets (2.29) as being multiplied with a "test function" and thus represents one equation for each choice of lapse, i.e. for each choice of foliation. The advantage of this viewpoint is that, although it may be impossible to solve the Hamilton-Jacobi equation for each choice of lapse, it may be possible to do so for specific choices. This may be enough in certain situations to draw interesting physical conclusions. One way or the other, it will be assumed in the following that a specific solution of (2.29) has been chosen and a specific spacetime with a specific foliation has been constructed from it.

It has been remarked (Kuchař, 1992) that the sandwich problem may present some obstacle in solving the Hamilton-Jacobi equation. This is because a complete solution to (2.29) can be interpreted as generating a canonical transformation between two three-geometries which are assumed to be connected by a single spacetime (which is in a sense "sandwiched" between these three-geometries). The uniqueness of the interpolating four-geometry was only proven in special cases, for example if $2 \rho-R>0$ (Belasco and Ohanian, 1969). It may thus happen that the complete solution to (2.29) cannot generate a canonical transformation between all three-geometries. In the semiclassical approximation, however, we use a special solution to (2.29) and can always construct a spacetime from a given three-geometry using the construction presented above.

Proceeding with the semiclassical expansion, one finds at order $M^{0}$ an 
equation involving also $S_{1}$ :

$$
G_{a b} \frac{\delta S_{0}}{\delta h_{a}} \frac{\delta S_{1}}{\delta h_{b}}-\frac{i \hbar}{2} G_{a b} \frac{\delta^{2} S_{0}}{\delta h_{a} \delta h_{b}}+\mathcal{H}_{m}=0
$$

As in the Klein-Gordon case, one can simplify this equation by introducing the wave functional

$$
\chi=D\left[h_{a}\right] \exp \left(i S_{1} / \hbar\right) .
$$

We will choose $D$ in such a way that the equations become simple. This is achieved by demanding

$$
G_{a b} \frac{\delta S_{0}}{\delta h_{a}} \frac{\delta D}{\delta h_{b}}-\frac{1}{2} G_{a b} \frac{\delta^{2} S_{0}}{\delta h_{a} \delta h_{b}} D=0,
$$

which can also be written in the form of a "conservation law"

$$
G_{a b} \frac{\delta}{\delta h_{a}}\left(\frac{1}{D^{2}} \frac{\delta S_{0}}{\delta h_{b}}\right)=0 .
$$

The interpretation of this equation becomes immediately clear if one writes down its pendant in one-dimensional quantum mechanics:

$$
\frac{\partial}{\partial x}\left(\frac{1}{D^{2}} \frac{\partial S_{0}}{\partial x}\right)=0 .
$$

This is nothing but the continuity equation for time-independent states. The solution of this simple equation gives the well known expression for the WKB prefactor $D \propto \sqrt{p}$.

Using (2.37) one finds from (2.34)

$$
i \hbar G_{a b} \frac{\delta S_{0}}{\delta h_{a}} \frac{\delta \chi}{\delta h_{b}} \equiv i \hbar \frac{\delta \chi}{\delta \tau}=\mathcal{H}_{m} \chi
$$

This is the functional Schrödinger equation in its local form (the TomonagaSchwinger equation) for quantum fields propagating on the classical spacetimes described by $S_{0}$. Note that the Schrödinger equation cannot be recovered if $S_{0}$ turns out to be a constant.

The above approach to semiclassical gravity has been discussed from various points of view by several authors including DeWitt (1967), Lapchinsky and Rubakov (1979), Banks (1985), Halliwell and Hawking (1985), Hartle (1986), and Kiefer (1987).

In $(2.38)$ one has introduced a time functional $\tau\left(x ; h_{a}\right]$ according to

$$
G_{a b}(x) \frac{\delta S_{0}}{\delta h_{a}(x)} \frac{\delta \tau\left(x ; h_{a}\right]}{\delta h_{b}(y)}=\delta(x-y) .
$$


This "many-fingered" time (which is also called WKB time) is defined on configuration space, but also yields a time parameter in each of the spacetimes described by $S_{0}$. This is clear from the above construction, since each spacetime is constructed from a specific foliation, which is labelled by $\tau$. It is for this reason that the "spacetime problem" (Isham, 1992; Kuchař, 1992) does not come into play in the semiclassical approximation. This problem refers to the fact that $\tau$ is not a spacetime scalar, i. e. that it depends on the embedding which is chosen. While this may in fact present a problem for approaches which attempt to use functions like $\tau$ as fundamental time functions in quantum gravity, there is no such ambiguity in the semiclassical approximation, where the spacetimes emerge with a specific foliation.

We also note that the hypersurfaces $\tau=$ constant in configuration space do not coincide with the hypersurfaces $S_{0}=$ constant. This can be immediately seen by comparing the definition of $\tau$ (2.39) with the Hamilton-Jacobi equation (2.29).

Another problem discussed in this context is the "global time problem." As simple minisuperspace models indicate, $\tau$ may not exist globally in the space of three-geometries. This is, however, not a real obstacle for the semiclassical approximation in that one can simply restrict the attention to regions in superspace where it exists. Since we will be interested in the following to quantum corrections only in the neighbourhood of a given spacetime this does not really present a restriction.

Barbour (1992) has stressed that the above introduced WKB time is very similar to the notion of ephemeris time as it is used by astronomers. This time is determined in retrospect from actual observations of the celestial bodies, exploiting the dynamical equations of the gravitational field. The above WKB time thus plays a double role: From one point of view one may simply interpret it as a coordinate on spacetime, but on the other side it is constructed from the geometry of a given three-dimensional space and is thus a quantity which in principle can be determined from astronomical observations. That ultimately the whole three-geometry is implemeted into the construction of this notion of time can very clearly be seen in the motion of the binary pulsar PSR $1913+16$ : If one attempts to determine ephemeris time from this system (exploiting the period decrease due to the radiation of gravitational waves) one reaches consistency only if the whole motion of the Galaxy and its "pull" on the binary pulsar is quantitatively taken into account (Damour and Taylor, 1991).

Up to this order, the total wave functional thus reads

$$
\Psi \approx \frac{1}{D} \exp \left(i M S_{0}\left[h_{a b}\right] / \hbar\right) \chi\left[h_{a b}, \phi\right]
$$

where $\chi$ obeys the Schrödinger equation (2.38). 


\subsection{Semiclassical gravity in the connection rep- resentation}

Instead of using the three-metric and is conjugate momentum as the basic variables one can perform a canonical transformation at the classical level to a set of new canonical variables consisting of the densitised triad and a complex $S O(3)$ connection. Details are presented in various other contributions to this volume. The fundamental equation is, of course, again of the form $H \Psi=0$, but the Hamiltonian reads differently, see Ashtekar (1991):

$$
\begin{aligned}
H= & \frac{G \hbar^{2}}{4 \pi} \epsilon^{i j k} F_{a b}^{k} \frac{\delta^{2}}{\delta A_{a}^{i} \delta A_{b}^{j}}+\hbar^{2} \frac{\delta^{2}}{\delta \phi^{2}}+2 G^{2} \hbar^{2} \partial_{a} \phi \partial_{b} \phi \frac{\delta^{2}}{\delta A_{a}^{i} \delta A_{b}^{i}} \\
& +\frac{\sqrt{2} \hbar^{3}}{3}\left(G^{3} m^{2} \phi^{2}+G^{2} \Lambda\right) \eta_{a b c} \epsilon^{i j k} \frac{\delta^{3}}{\delta A_{a}^{i} \delta A_{b}^{j} \delta A_{c}^{k}},
\end{aligned}
$$

where $F_{a b}^{k}=\partial_{a} A_{b}^{k}-\partial_{b} A_{a}^{k}-\epsilon^{k l m} A_{a}^{l} A_{b}^{m}$ is the field strength tensor associated with the complex connection $\mathbf{A}$, and $\eta_{a b c}$ is the metric-independent totally skew-symmetric density of weight -1 . I have used here a rescaled connection $A_{a}^{i} \equiv G \bar{A}_{a}^{i}$, where $\bar{A}_{a}^{i}$ denotes the connection as it is used in Ashtekar (1991). Such a rescaling is convenient for performing the semiclassical expansion and has also been proven useful in the investigation of the strong gravity limit, see Ashtekar (1988). The distinguished property of (2.41) is the fact that the potential term proportional to $R$ has been "swallowed" by the new variables. In addition, it contains only terms with functional derivatives (even third order derivatives) and that it is intrinsically complex since $\mathbf{A}$ is complex. Note also that a factor of $G$ is associated with each functional derivative with respect to $A_{a}^{i}$ which comes with a term containing $\phi$.

The semiclassical expansion now proceeds analogously to the geometrodynamical case of the previous section (the parameter $M$ is now just the inverse of $G$ ). The functional $S_{0}$ does not depend on non-gravitational fields, and it obeys the Hamilton-Jacobi equation

$$
\frac{\epsilon^{i j k}}{4 \pi} F_{a b}^{k} \frac{\delta S_{0}}{\delta A_{a}^{i}} \frac{\delta S_{0}}{\delta A_{b}^{j}}+\frac{i \sqrt{2}}{3} \Lambda \eta_{a b c} \epsilon_{i j k} \frac{\delta S_{0}}{\delta A_{a}^{i}} \frac{\delta S_{0}}{\delta A_{b}^{j}} \frac{\delta S_{0}}{\delta A_{c}^{k}}=0 .
$$

Note that since the momentum conjugate to $A_{a}^{i}, \tilde{E}_{i}^{a}$, is replaced by $\delta / \delta A_{a}^{i}$ (without an $i$ ) in the Schrödinger representation, the momentum is given by $\tilde{E}_{i}^{a}=i \delta S_{0} / \delta A_{a}^{i}$. The triad $\tilde{E}_{i}^{a}$ is not necessarily real, but it can be made real since it satisfies Gauss's law. Note that $S_{0}=$ constant is always a solution of this equation.

The next order $\left(G^{0}\right)$ yields the functional Schrödinger equation for the wave functional $\chi \equiv D e^{i S_{1}}$,

$$
i \hbar \frac{\epsilon^{i j k}}{4 \pi} F_{a b}^{k} \frac{\delta S_{0}}{\delta A_{a}^{i}} \frac{\delta \chi}{\delta A_{b}^{j}} \equiv i \hbar \frac{\delta \chi}{\delta \tau}=\tilde{H}_{m} \chi
$$


where the Hamiltonian density $\tilde{H}_{m}$ is now given by the expression

$$
\begin{aligned}
\tilde{H}_{m}= & -\frac{\hbar^{2}}{2} \frac{\delta^{2}}{\delta \phi^{2}}+\frac{\delta S_{0}}{\delta A_{a}^{i}} \frac{\delta S_{0}}{\delta A_{b}^{i}} \partial_{a} \phi \partial_{b} \phi+\frac{i}{3 \sqrt{2}} m^{2} \phi^{2} \eta_{a b c} \epsilon_{i j k} \frac{\delta S_{0}}{\delta A_{a}^{i}} \frac{\delta S_{0}}{\delta A_{b}^{j}} \frac{\delta S_{0}}{\delta A_{c}^{k}} \\
& +\sqrt{2} \Lambda \hbar \eta_{a b c} \epsilon_{i j k} \frac{\delta S_{0}}{\delta A_{a}^{i}}\left(\frac{\delta S_{0}}{\delta A_{b}^{j}} \frac{\delta}{\delta A_{c}^{k}}-\frac{1}{D} \frac{\delta S_{0}}{\delta A_{b}^{j}} \frac{\delta D}{\delta A_{c}^{k}}+\frac{\delta^{2} S_{0}}{\delta A_{b}^{j} \delta A_{c}^{k}}\right) .
\end{aligned}
$$

A comparison with (2.41) exhibits that $\tilde{H}_{m}$ is equal to $H$ as evaluated on the classical gravitational background determined by the Hamilton-Jacobi equation except for the last three $\Lambda$ - dependent terms in (2.44) which arise due to the presence of the third functional derivatives in (2.41). These terms can be absorbed by a redefinition of the wave functional, but their interpretation is not yet clear.

It seems that the traditional semiclassical approximation is tied to the presence of only second order derivatives (i.e. quadratic momenta) in the Hamiltonian. Consider, for example, the anharmonic oscillator in quantum mechanics, which is defined by the Hamiltonian

$$
H=\frac{p^{2}}{2}+\lambda x^{4} \text {. }
$$

If one wished to use the momentum representation instead of the position representation for the wave function in the Schrödinger equation, one would have to substitute fourth order derivatives in the momentum for the second term in $H$. Performing a WKB expansion one finds terms analogous to the ones in (2.41), which one would have to absorb through a redefinition of the wave function to get a transparent interpretation. This problem reflects of course the unsolved issue of the Hilbert space structure of the full theory (which would allow much less states than the number of solutions to $H \Psi=$ $0)$.

A second major difference to the geometrodynamical case is the complexity of the connection, as mentioned above. One has been able to write (2.43) as a functional Schrödinger equation after, in an appropriate region of configuration space, a time functional $\tau(\mathbf{x} ; \mathbf{A}]$ was introduced, which satisfies

$$
\delta(\mathbf{x}-\mathbf{y})=\frac{\epsilon^{i j k}}{4 \pi} F_{a b}^{k}(\mathbf{y}) \frac{\delta S_{0}}{\delta A_{a}^{i}(\mathbf{y})} \frac{\delta \tau(\mathbf{x} ; \mathbf{A}]}{\delta A_{b}^{j}(\mathbf{y})} .
$$

This time functional, however, is complex and thus not viable as a semiclassical time parameter. Since one generally assumes that the functionals in the connection representation are holomorphic, one can take the real part of $\tau$ as a candidate for WKB time.

Since one wants to recover real quantum gravity at a certain stage, one must implement appropriate reality conditions (Ashtekar, 1991). The assumption, which is motivated by a comparison with the Bargmann representation for the harmonic oscillator, is that they are used to determine the 
inner product which has to be imposed on the physical states. The present semiclassical approximation, however, only works on the level of the differential equation and therefore does not "know" about the Hilbert structure (see the remarks above). It may even be possible that both concepts are in conflict with each other, i. e. that the semiclassical approximation "runs out" of the Hilbert space (Louko, 1993). It is definitely not possible to implement the reality conditions on the wave functional in the same way as the constraint equation. One may therefore be able to decide the compatibility only after the appropriate Hilbert space structure of the full theory has been determined.

The above derivation has to be contrasted with the derivation of the Schrdinger equation by Ashtekar (1991). He expands the classical Hamiltonian constraint up to second order around some given classical configuration, which is chosen to be a flat triad on a non compact manifold. The important feature of the truncated constraint is its linearity in the triad, i. e. in the canonical momentum. As in the full theory, quantisation proceeds by implementing the truncated constraint as a condition on wave functionals. The result is

$$
\begin{aligned}
& -\hbar \frac{1}{G}\left(\Delta \frac{\delta}{\delta A^{T}(x)}\right) \Psi\left(A^{T T}, A^{L}, A^{T}, A^{A}\right) \\
& =G A^{T T a b}(x)\left(A_{a b}^{T T}(x)\right)^{*} \Psi\left(A^{T T}, A^{L}, A^{T}, A^{A}\right) .
\end{aligned}
$$

The internal indices have here all been converted to space indices by the use of the given background triad, and we have decomposed the resulting "connection tensor" into its transverse-traceless, longitudinal, trace, and antisymmetric parts.

The important step is now to identify the term $G(\Delta)^{-1} A^{T}(x)$ as a "manyfingered" parameter $\tau(x)$, which has the dimension of time. (This is a nonlocal expression in the connection.) As in the above semiclassical expansion, it is, however, complex and thus not suitable as a physical time. But, again, assuming the holomorphicity of the wave functional one can identify its imaginary part as physical time, since then the left-hand side of (2.46) reads

$$
-\hbar \frac{\delta}{\delta \tau(x)} \Psi=i \hbar \frac{\delta}{\delta(\operatorname{Im} \tau)(x)} \Psi,
$$

while the right-hand side is the "physical" Hamiltonian in this order, i. e. it generates the dynamics of the transverse traceless degrees of freedom (the "gravitons"). One has thus recovered, in the weak field approximation, the Schrödinger equation from quantum gravity.

It was important in the above derivation that the classical constraint has been expanded up to quadratic order in the deviation of the fields from their flat space values, since the left-hand side of (2.46) would have been zero in the 
first order approximation. This has already been recognised by Kuchař (1970) in his analysis of the weak field limit in the geometrodynamical language: The linear gravitons do not contain "information about time." This information shows first up in the gravitational field which they produce in the next order. Ashtekar (1991) has related an intrinsic degree of freedom in configuration space, the imaginary part of the trace part of the connection, to physical time. In the geometrodynamical language, this imaginary part is basically given by the trace of the extrinsic curvature and is thus an "extrinsic time." Not surprisingly, this was the time parameter chosen by Kuchař (1970), who gave a lucid geometric interpretation: Consider a hypersurface in flat spacetime by specifying $t$ as a function of $x^{i}$. If this hypersurface is attained from a flat hypersurface by a small deformation, Kuchař showed that the intrinsic geometry is only sensitive to the second order in this deformation $\left(h_{i j} \approx\right.$ $\left.\delta_{i j}+t,{ }_{i} t,{ }_{j}\right)$, while the extrinsic geometry is already sensitive to the first order $\left(K_{i k} \approx t,{ }_{i k}\right)$ and thus seems to be more appropriate in defining a time variable.

What are the main differences of this approach to the semiclassical expansion, which was presented above? One difference is that, whereas above the whole gravitational field was semiclassical in the first order, in the Ashtekar approach some of the degrees of freedom are treated as fully quantum, i. e. they appear in the Hamiltonian in the Schrödinger equation. This is, however, not a major difference since, as has been remarked in the previous section, one could easily generalise the scheme to incorporate such a situation by expanding with respect to a parameter different from $G$. It is thus not surprising that Ashtekar's definition of time contains the gravitational constant.

What is more important is that in the second approach a classical background has been chosen $a b$ initio and only a truncated constraint, being linear in the canonical momentum, has been quantised. It is far from clear whether the same results can be recovered from the full theory in an appropriate limit.

Whereas time in the semiclassical approximation is constructed from the phase of the WKB state and thus essentially state-dependent, the weak field approach constructs time always from the same part of the configuration variables and is thus state-independent. Moreover, the semiclassical limit seems to be inappropriate for studying the weak field limit, since a flat background spacetime in its standard foliation corresponds to $S_{0}=$ constant ("no change of configuration" $\leftrightarrow$ "no WKB time") and thus does not allow the recovery of a Schrödinger equation in this case. This difficulty of incorporating "flat spacetime" into the picture of quantum geometrodynamics will again show up in the next order of this approximation scheme. 


\section{Chapter 3}

\section{Corrections to the Schrödinger equation from quantum gravity}

\subsection{General derivation and discussion}

We continue with the semiclassical expansion scheme to the order $M^{-1}$, which leads to the calculation of correction terms to the functional Schrödinger equation (2.38). This order yields an equation involving $S_{2}$ (compare (2.27)) and reads explicitly

$$
\begin{aligned}
& G_{a b} \frac{\delta S_{0}}{\delta h_{a}} \frac{\delta S_{2}}{\delta h_{b}}+\frac{1}{2} G_{a b} \frac{\delta S_{1}}{\delta h_{a}} \frac{\delta S_{1}}{\delta h_{b}}-\frac{i \hbar}{2} G_{a b} \frac{\delta^{2} S_{1}}{\delta h_{a} \delta h_{b}} \\
& +\frac{1}{\sqrt{h}}\left(\frac{\delta S_{1}}{\delta \phi} \frac{\delta S_{2}}{\delta \phi}-\frac{i \hbar}{2} \frac{\delta^{2} S_{2}}{\delta \phi^{2}}\right)=0 .
\end{aligned}
$$

One first rewrites this equation by substituting $S_{1}$ in favour of $\chi$, see (2.35), and then makes the following ansatz for $S_{2}$ :

$$
S_{2}\left[h_{a}, \phi\right]=\sigma_{2}\left[h_{a}\right]+\eta\left[h_{a}, \phi\right] .
$$

Eq. (3.1) will be simplified, if one demands that $\sigma_{2}$ obeys:

$$
G_{a b} \frac{\delta S_{0}}{\delta h_{a}} \frac{\delta \sigma_{2}}{\delta h_{b}}-\frac{\hbar^{2}}{D^{2}} G_{a b} \frac{\delta D}{\delta h_{a}} \frac{\delta D}{\delta h_{b}}+\frac{\hbar^{2}}{2 D} G_{a b} \frac{\delta^{2} D}{\delta h_{a} \delta h_{b}}=0 .
$$

The interpretation of this equation becomes immediately clear, if one writes out its analogue in one-dimensional quantum mechanics:

$$
\sigma_{2}^{\prime}=-\frac{\hbar^{2}}{4} \frac{p^{\prime \prime}}{p^{2}}+\frac{3 \hbar^{2}}{8} \frac{p^{2}}{p^{3}}
$$

where, again, $p=\partial S / \partial x \equiv S^{\prime}$. Eq. (3.4) is nothing but the equation for the second-order WKB correction, see e. g. Landau and Lifshitz (1975). 
(Recall that for pure gravity an expansion in $G$ is fully equivalent to an expansion in $\hbar$.) The separation of $\sigma_{2}$ from $S_{2}$ thus serves to separate the pure gravitational $\mathrm{WKB}(2)$ factor (which in this context is uninteresting) from the relevant part, which satisfies the corrected Schrödinger equation. We then find from (3.1) an equation involving $\eta$ only:

$$
\begin{aligned}
G_{a b} \frac{\delta S_{0}}{\delta h_{a}} \frac{\delta \eta}{\delta h_{b}}= & \frac{\hbar^{2}}{2 \chi}\left(-\frac{2}{D} G_{a b} \frac{\delta \chi}{\delta h_{a}} \frac{\delta D}{\delta h_{b}}+G_{a b} \frac{\delta^{2} \chi}{\delta h_{a} \delta h_{b}}\right) \\
& +\frac{i \hbar}{\sqrt{h} \chi} \frac{\delta \eta}{\delta \phi} \frac{\delta \chi}{\delta \phi}+\frac{i \hbar}{2 \sqrt{h}} \frac{\delta^{2} \eta}{\delta \phi^{2}} .
\end{aligned}
$$

Up to this order, the total wave functional is thus of the form

$$
\Psi=\frac{1}{D} \exp \left(\frac{i}{\hbar}\left(M S_{0}+\sigma_{2} M^{-1}\right)\right) \chi \exp \left(\frac{i \eta}{M \hbar}\right) .
$$

The wave functional

$$
\psi \equiv \chi \exp \left(\frac{i \eta}{M \hbar}\right)
$$

then obeys the "corrected Schrödinger equation" (Kiefer and Singh, 1991)

$$
i \hbar \frac{\delta \psi}{\delta \tau}=\mathcal{H}_{m} \psi+\frac{h^{2}}{M \chi} G_{a b}\left(\frac{1}{D} \frac{\delta D}{\delta h_{a}} \frac{\delta \chi}{\delta h_{b}}-\frac{1}{2} \frac{\delta^{2} \chi}{\delta h_{a} \delta h_{b}}\right) \psi
$$

Thus, if one knows the solutions to the previous order equations, one can evaluate the correction term on the right-hand side of (3.8). Note that it is not sufficient to know the solution, $\chi$, of the Schrödinger equation (2.38), but that the knowledge of the gravitational prefactor, $D$, is also required.

As in the case of the uncorrected Schrödinger equation, Eq. (3.8) is equivalent to its integrated form, since a specific slicing of a specific spacetime has been chosen upon giving a solution to the Hamilton-Jacobi equation $(2.29)$.

To write (3.8) in a more transparent way, we decompose the vector fields on the right-hand side of (3.8) into their respective components tangential and normal to the integral curves of the vector field $G_{a b} \delta S_{0} / \delta h_{a}$. This will enable us to use the Schrödinger equation (2.38) for the tangential part. We first decompose the first derivative according to

$$
G_{a b} \frac{\delta \chi}{\delta h_{a}}=\alpha G_{a b} \frac{\delta S_{0}}{\delta h_{a}}+a_{b}
$$

where

$$
\frac{\delta S_{0}}{\delta h_{a}} a_{a}=0
$$


The coefficient $\alpha$ is determined by multiplying each side of (3.9) with $\delta S_{0} / \delta h_{b}$, summing over $b$, and making use of the Hamilton-Jacobi equation (2.29) as well as the Schrödinger equation (2.38). One finds

$$
\alpha=\frac{i}{2 \hbar V} \mathcal{H}_{m} \chi
$$

This, of course, holds only if the potential $V$ is non-vanishing, i. e. in regions where the vector field $G_{a b} \delta S_{0} / \delta h_{a}$ is not "lightlike." (Note that in the general case $V$ is nonzero, since it also includes contributions from "macroscopic" matter sources.) In the "lightlike" case $\alpha$ cannot be unambiguously determined, since the addition of a "lightlike" vector on the right-hand side of (3.9) does not change the scalar product of the left-hand side with $\delta S_{0} / \delta h_{b}$. But it is only this scalar product which is determined by the previous order equation (2.38).

I emphasise that the component $a_{b}$ is not determined by the previous order equations, but only by the boundary conditions which are imposed on the full wave functional $\Psi$. As can be explicitly seen from (3.8), the second derivatives with respect to the three-metric now come into play. We will assume in the following that these component can be neglected, i. e. we will choose the boundary conditions such that $\chi$ is "peaked" around the considered worldline in configuration space, i. e. along the particular spacetime we have chosen (Cosandey, 1993).

The second derivative term on the right-hand side of (3.8) is now also decomposed into its tangential and normal components, and use of the previous order equations as well as of (3.10) is made. After some calculation one can then put (3.8) in the form (Kiefer and Singh, 1991)

$$
i \hbar \frac{\delta \psi}{\delta \tau}=\mathcal{H}_{m} \psi+\frac{4 \pi G}{c^{4} \sqrt{h}(R-2 \Lambda)} \mathcal{H}_{m}^{2} \psi+i \hbar \frac{4 \pi G}{c^{4}} \frac{\delta}{\delta \tau}\left(\frac{\mathcal{H}_{m}}{\sqrt{h}(R-2 \Lambda)}\right) \psi .
$$

This is the central equation of this section. Several comments are in order.

(1) The WKB(1) prefactor $D$ has disappeared from the corrected Schrödinger equation after use of (2.36) was made. This is fortunate, since we do not have to deal with the pure gravitational part of the full wave functional.

(2) As remarked above, this form of the corrected Schrödinger equation only holds for non-vanishing gravitational potential. In the general case one must resort to the form (3.8).

(3) The correction terms in (3.11) are, of course, only formal as long as no regularisation scheme is brought into play to cure the divergences which come together with the functional derivatives. We will show in the section on applications how concrete physical prediction can nevertheless be extracted from these terms in special cases.

(4) The terms in (3.11) are independent of the factor ordering which is chosen for the gravitational kinetic term in (2.1). There may, however, be an ambiguity in the factor ordering of the gravity-matter coupling. 
(5) If "macroscopic" matter sources are present, one has to substitute $\sqrt{h}(R-2 \Lambda)$ in the denominator of $(3.11)$ by $\sqrt{h}(R-2 \Lambda)-\epsilon_{m} / 2 M c^{2}$, where $\epsilon_{m}$ is an effective matter energy density. That $M \propto G^{-1}$ comes explicitly into play in this expression, does not present any problem as long as the corresponding correction terms are small. This will become important in the example of black hole evaporation discussed below.

(6) In addition to the Wheeler-DeWitt equation (2.1) one must also expand the momentum constraints. This is straightforward and leads to the result that the wave functional remains unchanged under a spatial diffeomorphism at each order of this approximation scheme.

(7) The second correction term in (3.11) is pure imaginary and thus leads to a violation of unitarity. The occurrence of such a term is not surprising, since we have attempted to write down an effective equation for the nongravitational fields alone. This can also be recognised from the point of view of the conservation laws for the wave functional. The full Wheeler-DeWitt equation (2.1) is of the Klein-Gordon type and thus obeys the following conservation law:

$$
\frac{1}{M} G_{a b} \frac{\delta}{\delta h_{a}}\left(\Psi^{*} \frac{\overleftrightarrow{\delta}}{\delta h_{b}} \Psi\right)+\frac{1}{\sqrt{h}} \frac{\delta}{\delta \phi}\left(\Psi^{*} \frac{\overleftrightarrow{\delta}}{\delta \phi} \Psi\right)=0
$$

Applying the $M$-expansion for the wave functional to this conservation law, one finds at the level of the corrected Schrödinger equation

$$
\begin{aligned}
& \frac{\delta}{\delta \tau}\left(\psi^{*} \psi\right)+\frac{\hbar}{2 i \sqrt{h}} \frac{\delta}{\delta \phi}\left(\psi^{*} \frac{\overleftrightarrow{\delta}}{\delta \phi} \psi\right) \\
& -\frac{i \hbar}{2 M} G_{a b}\left[\frac{\delta}{\delta h_{a}}\left(\psi^{*} \frac{\overleftrightarrow{\delta}}{\delta h_{b}} \psi\right)-\frac{1}{D} \frac{\delta D}{\delta h_{a}} \psi^{*} \frac{\delta}{\delta h_{b}} \psi\right]=0
\end{aligned}
$$

The first two terms together are the "Schrödinger current" connected with (2.38), while the remaining terms yield correction terms proportional to $M^{-1}$. Upon functionally integrating this equation over the field $\phi$ and making the standard assumption that $\psi$ falls off for large field configurations, one finds from (3.13)

$$
\frac{d}{d t} \int \mathcal{D} \phi \psi^{*} \psi=\frac{8 \pi G}{c^{4}} \int \mathcal{D} \phi \int d^{3} x \psi^{*} \frac{\delta}{\delta \tau}\left(\frac{\mathcal{H}_{m}}{\sqrt{h}(R-2 \Lambda)}\right) \psi
$$

A comparison with (3.11) immediately exhibits that the violation of the Schrdinger conservation law produces the imaginary correction term in (3.11). I note again that a similar term emerges in the nonrelativistic expansion of the Klein-Gordon equation in the presence of an external Newtonian potential (Lämmerzahl, 1993). 
Corrections to the Schrödinger equation have been discussed, in the context of one-dimensional minisuperspace models, by a number of authors including Brout and Venturi (1989), Singh (1990), Kowalski-Glikman and Vink (1990), and Bertolami (1991) (see also Singh (1993) for a review). The above, systematic, expansion in the context of the full Wheeler-DeWitt equation has been discussed by Kiefer and Singh (1991).

The above semiclassical expansion scheme can also be applied to standard quantum field theories like QED, see Kiefer, Padmanabhan, and Singh (1991), and Kiefer (1992a). The purpose of such an application lies primarily in the test of its viability within a well-understood framework. It provides, however, also a useful insight into the semiclassical expansion of QED itself. It demonstrates, for example, that one can start from the stationary equation for full QED and derive, as in the gravitational case, a time-dependent Schrödinger equation for the matter fields in an external electromagnetic background. Again, the "fast" matter degrees of freedom follow the "slow" electromagnetical degrees of freedom, which can thus serve as an "intrinsic clock."

Formally, the expansion is performed with respect to the electric charge (after a suitable rescaling of the vector potential has been made). The expression for the full wave functional up to order $e^{0}$ then reads, in analogy to the gravitational expression (2.40),

$$
\Psi \approx \frac{1}{D} \exp \left(\frac{i S_{0}[\mathbf{A}]}{\hbar e^{2}}\right) \psi\left[\mathbf{A}, \phi, \phi^{\dagger}\right]
$$

where $\mathbf{A}$ is the vector potential, and $\phi$ is a charged scalar field. The functional $\psi$ obeys a functional Schrödinger equation, in which time is defined by $S_{0}$. Corrections to this Schrödinger equation can be found in order $e^{2}$ and look very similar to the gravitational terms in (3.11). In QED they can be easily interpreted in terms of Feynman diagrams with an internal photon line, see Kiefer (1992a). This in turn provides a bridge to the standard expansion of the effective action.

I want to conclude this section with a brief remark on the correction terms in the Ashtekar variables. Basically, the expansion proceeds as in the geometrodynamical case. The main difference is that there is no $R$ term in the analogue of (3.11), since the potential $V$ in the Ashtekar case is given by the cosmological constant and the macroscopic matter contribution only.

\subsection{Applications}

\subsubsection{A minisuperspace example}

The simplest example is to neglect all inhomogeneous degrees of freedom and keep only homogeneous ones like the scale factor $a$ of a (closed) Fried- 
mann Universe and a homogeneous scalar field $\phi$. The analogue of (3.11) is then a quantum mechanical Schrödinger equation for a wave function $\psi(a, \phi)$ augmented by two correction terms:

$$
i \hbar \dot{\psi}=H_{m} \psi+\frac{G}{3 \pi c^{4} a}\left(H_{m}^{2}+i \hbar H_{0}\left[-\frac{\partial H_{m}}{a \partial a}+H_{m}\right]\right) \psi
$$

Here, $H_{0} \equiv \dot{a} / a$ denotes the Hubble parameter of the Friedmann Universe, and $H_{m}$ is the Hamiltonian for the scalar field. One recognises that the correction terms only become relevant for small scales $(a \rightarrow 0)$, i. e. in the early Universe.

\subsubsection{Scalar field in de Sitter space}

As a specific example we treat the case of a minimally coupled scalar field, whose Hamiltonian density is given by $(2.25)$ with $U \equiv 0$. The case of nonminmally fields involves extra subtleties and is treated elsewhere, see Kiefer (1993b). It is convenient to write the three-metric in the following form:

$$
h_{a b}=h^{1 / 3} \tilde{h_{a b}}
$$

where $h$ is the determinant of the three-metric. (The "conformal part" $\tilde{h_{a b}}$ thus has unit determinant.) The Hamilton-Jacobi equation (2.29) then reads

$$
-\frac{3 \sqrt{h}}{16}\left(\frac{\delta S_{0}}{\delta \sqrt{h}}\right)^{2}+\frac{\tilde{h_{a c}} \tilde{h_{b d}}}{2 \sqrt{h}} \frac{\delta S_{0}}{\delta h_{a b}} \frac{\delta S_{0}}{\delta \tilde{h_{c d}}}-2 \sqrt{h}(R-2 \Lambda)=0
$$

We want to look for a solution of this equation which, after integrating (2.30), describes de Sitter spacetime in a flat foliation. This is easily achieved by setting $R=0$ in (3.18) and looking for a solution of the form $S_{0}=S_{0}(\sqrt{h})$. The desired solutions read

$$
S_{0}= \pm 8 \sqrt{\frac{\Lambda}{3}} \int \sqrt{h} d^{3} x \equiv \pm 8 H_{0} \int \sqrt{h} d^{3} x
$$

where, again, $H_{0}$ is the Hubble parameter (which is a constant in this case). According to $(2.38), S_{0}$ defines a local time parameter in configuration space,

$$
\frac{\delta}{\delta \tau}=-\frac{3 \sqrt{h}}{8} \frac{\delta S_{0}}{\delta \sqrt{h}} \frac{\delta}{\delta \sqrt{h}}=\sqrt{3 h \Lambda} \frac{\delta}{\delta \sqrt{h}},
$$

from where the well kown expansion law for the scale factor can be found,

$$
\frac{\partial a^{3}}{\partial t}=\int d^{3} y \frac{\delta \sqrt{h}(\mathbf{x})}{\delta \tau(\mathbf{y})}=3 H_{0} a^{3} \rightarrow a(t)=e^{H_{0} t} .
$$


Equation (2.36) for the prefactor can be immediately solved and yields $\delta D / \delta \tau=$ 0 , i. e. $D$ is constant on the whole de Sitter spacetime. The functional Schrödinger equation (2.38) then reads in its integrated form (setting $\hbar=1$ in the following)

$$
i \dot{\psi}=\int d^{3} x\left(-\frac{1}{2 a^{3}} \frac{\delta^{2}}{\delta \phi^{2}}+\frac{a}{2}(\nabla \phi)^{2}+\frac{a^{3}}{2} m^{2} \phi^{2}\right) \psi .
$$

Since de Sitter space is homogeneous, it is adequate to use a momentum representation, i. e.

$$
\phi(\mathbf{x})=\int \frac{d^{3} k}{(2 \pi)^{3}} \chi(\mathbf{k}) e^{i \mathbf{k x}} \equiv \int d \tilde{\mathbf{k}} \chi_{k} e^{i \mathbf{k x}},
$$

and thus

$$
\frac{\delta}{\delta \phi(\mathbf{x})}=\int d \tilde{k} e^{i \mathbf{k x}} \frac{\delta}{\delta \chi_{k}} ; \frac{\delta \chi_{k}}{\delta \chi_{k^{\prime}}}=(2 \pi)^{3} \delta^{(3)}\left(\mathbf{k}+\mathbf{k}^{\prime}\right)
$$

We want to solve (3.21) by a Gaussian ansatz, i. e.

$$
\psi=N(t) \exp \left(-\frac{1}{2} \int d \tilde{k} \Omega(\mathbf{k}, t) \chi_{k} \chi_{-k}\right) .
$$

Gaussian states are used to describe generalised vacuum states in the Schrödinger picture, see e. g. Jackiw (1988b). This special form is of course tied to quadratic Hamiltonians like (2.25) with $U=0$. According to (3.21), the Gaussian form (3.23) is preserved in time. Inserting this ansatz into (3.21) yields two equations for $N$ and for $\Omega$ :

$$
\begin{aligned}
i \frac{\dot{N}}{N} & =\frac{1}{2 a^{3}} \operatorname{Tr} \Omega \equiv \frac{V}{2 a^{3}} \int d \tilde{k} \Omega(\mathbf{k}, t) \\
i \dot{\Omega} & =\frac{\Omega^{2}}{a^{3}}-a^{3}\left(m^{2}+\frac{k^{2}}{a^{2}}\right) .
\end{aligned}
$$

Eq. (3.25) can be simplified by writing

$$
\Omega=-i a^{3} \frac{\dot{y}}{y}
$$

which leads to a linear second-order equation for $y$,

$$
\ddot{y}+3 H_{0} \dot{y}+\left(m^{2}+\frac{k^{2}}{a^{2}}\right) y=0 .
$$

It is useful to introduce a conformal time coordinate $\eta$,

$$
d t=a d \eta \rightarrow a(\eta)=-\frac{1}{H_{0} \eta}, \eta \in(0,-\infty),
$$


which yields

$$
y^{\prime \prime}+2 \frac{a^{\prime}}{a} y^{\prime}+\left(m^{2} a^{2}+k^{2}\right) y=0
$$

where ' denotes a derivative with respect to $\eta$. Inserting the evolution law (3.28) for the scale factor, this equation can be explicitly solved in terms of Hankel functions, see Guven, Lieberman, and Hill (1989). The question then arises what boundary conditions one has to choose to select a particular solution of (3.29). We will follow Guven et al and choose the Bunch-Davies vacuum. This is de Sitter invariant, i. e. invariant under $S O(3,1)$, and reduces to the Minkowski vacuum at early times, i. e. $\Omega$ tends to $\sqrt{k^{2}+m^{2}}$ as $t \rightarrow-\infty$, where the metric is essentially static and one can put $\dot{\Omega}=0$ and $a=1$ in (3.25). The demand for de Sitter invariance is the analogue of the demand for Poincaré invariance in Minkowski space. In $2+1$ dimensions it has been shown that the Bunch-Davies vacuum is the only de Sitter invariant vacuum state, see Jackiw (1988b).

We are now interested to calculate the expectation value of the Hamiltonian density with respect to (3.23). Using the solution for $\Omega$ which corresponds to the Bunch-Davies vacuum, one finds for $\left\langle\phi^{2}\right\rangle$,

$$
\left\langle\phi^{2}\right\rangle=\int \frac{d \tilde{k}}{2 \operatorname{Re} \Omega} \propto \frac{H_{0}^{2}}{4 \pi^{2}} \int_{0}^{\infty} d y y
$$

i. e. a divergent result! The occurrence of ultraviolet divergencies is of course not surprising, and we need a regularisation scheme to extract physical predictions from (3.23). Guven et al (1989) employ a dimensional regularisation scheme: They evaluate all expressions in $d$ space dimensions, where they are finite, and subtract terms which diverge in the limit $d \rightarrow 3$. After some lengthy calculation they find a finite result for the expectation value of the Hamiltonian which agrees with the result found by using the effective action. In the massless case, for example, one has (re-inserting $\hbar$ and $c$ )

$$
\left\langle\mathcal{H}_{m}\right\rangle=\frac{29 \hbar H_{0}^{4} a^{3}}{960 \pi^{2} c^{3}} .
$$

This result is obtained on the level of the functional Schrödinger equation (3.21), which is the level of quantum field theory on a classical spacetime.t As we have seen in the last section, the next order of the semiclassical approximation scheme yields corrections to the Schrödinger equation. In the present case, Eq. (3.11) reads

$$
i \hbar \frac{\delta \psi}{\delta \tau}=\mathcal{H}_{m} \psi-\frac{2 \pi G}{c^{4} \sqrt{h} \Lambda} \mathcal{H}_{m}^{2} \psi-i \hbar \frac{2 \pi G}{c^{4} \Lambda} \frac{\delta}{\delta \tau}\left(\frac{\mathcal{H}_{m}}{\sqrt{h}}\right) \psi .
$$

\footnotetext{
${ }^{1}$ Note that $\left\langle\mathcal{H}_{m}\right\rangle=a^{3}\left\langle T_{00}\right\rangle$, where $T_{00}$ is the 00- component of the energy-momentum tensor.
} 
The first correction term in (3.31) leads to a shift in the above expectation value, while the second term is a source of non-unitarity. I want to focus first on the real term. It causes a shift

$$
\left\langle\mathcal{H}_{m}\right\rangle \rightarrow\left\langle\mathcal{H}_{m}\right\rangle-\frac{2 \pi G}{3 c^{2} a^{3} H_{0}^{2}}\left\langle\mathcal{H}_{m}^{2}\right\rangle
$$

The last term is of course utterly divergent and it is not clear how to regularise it. On the covariant level it corresponds to a term like $\left\langle T_{\mu \nu}^{2}(x)\right\rangle$, i. e. to a four-point correlation function, whose regularisation in the presence of gravitational fields has, to my knowledge, not been addressed. Nevertheless, one can evaluate (3.32) in the present case, at least in a heuristic sense. The reason is that the Bunch-Davies vacuum state is an adiabatic vacuum state. The fluctuation of such a state would be zero in quantum mechanics. I assume that this holds also for the present field theoretic case, i. e. that

$$
\left\langle\mathcal{H}_{m}^{2}\right\rangle \approx\left\langle\mathcal{H}_{m}\right\rangle^{2}
$$

The covariant version of (3.33) is frequently employed as a criterion for semiclassical behaviour (Kuo and Ford, 1993). This will be discussed in more detail in the next section.

One thus gets the following prediction for the shift in the expectation value (3.30):

$$
\left\langle\mathcal{H}_{m}\right\rangle \rightarrow\left\langle\mathcal{H}_{m}\right\rangle-\frac{841}{1382400 \pi^{3}} \frac{G \hbar H_{0}^{6} a^{3}}{c^{8}} .
$$

Although found by using heuristic arguments, this is a definite prediction of the Wheeler-DeWitt equation! That the correction term is proportional to $G H_{0}^{6}$ is of course clear from dimensional arguments. We remark already at this point that there will be a second contribution to the energy shift which arises from the modification of semiclassical time through the back reaction of the matter fields. Surprisingly, this modification will just lead to a change of sign in the energy shift (3.34). The details will be presented in the next section.

What about the unitarity violating term? Again, its regularisation is unclear, but one can make the following rough estimate. It produces an imaginary contribution to the energy density of the order of magnitude

$$
\begin{aligned}
|\operatorname{Im} \epsilon| & =\frac{2 \pi G \hbar}{3 c^{2} H_{0}^{2}} \frac{\left\langle\mathcal{H}_{m}\right\rangle}{\tau V a^{3}} \\
& \approx \frac{2 \pi G \hbar}{c^{2} H_{0} V} \frac{\left\langle\mathcal{H}_{m}\right\rangle}{a^{3}}=\frac{29 G \hbar^{2} H_{0}^{3}}{480 \pi V c^{5}} .
\end{aligned}
$$

The presence of an energy density with an imaginary part is of course an indication of a possible instability of the system. The associated time scale 
$t^{*}$ on which this "quantum gravitational instability of de Sitter space" may become relevant is given by

$$
t^{*}=(2 \operatorname{Im} \epsilon V)^{-1} \hbar=\frac{240 \pi c^{5}}{29 G \hbar H_{0}^{3}} \sim\left(\frac{H_{0}^{-1}}{t_{P l}}\right)^{3},
$$

where $t_{P l}$ is the Planck length. This time scale is thus only relevant if the "horizon scale" $H_{0}^{-1}$ is of the order of the Planck length.

\subsubsection{Evaporation of black holes}

We now turn to an example where the second, imaginary, correction term in (3.11) becomes important. This will be the case if the mass of a black hole approaches the Planck regime through the emission of Hawking radiation (Kiefer, Müller, and Singh, 1993).

A black hole is produced by the collapse of matter, which we can assume for simplicity to be in a pure state initially. The presence of quantum fields on the black hole spacetime leads to Hawking radiation, i. e. the black hole emits thermal radiation and shrinks. What happens at the final stage of this evaporation is an open issue. If the evaporation is complete, only the Hawking radiation is left behind. Since thermal radiation cannot, however, be described by a pure state, there seems to be an evolution from pure states into mixed states. This was taken as a hint that quantum gravity may violate unitarity (Hawking, 1976) and that information is lost at a fundamental level. There are, however, suggestions how this "information loss paradox" can be resolved (a recent review of this issue is Page (1993)). One possibility is that the prescription through Hawking radiation is incomplete and that the true time evolution is unitary. This would mean that information must be encoded in the black hole radiation, for example by stimulated emission or correlations. A second possibility is that the black hole evaporation is not complete but ceases at a mass of the order of the Planck mass, leaving behind a Planck mass remnant. The main problem with this approach is that only little energy is available to store a huge amount of information.

The main drawback of all these approaches is that, although the problem is a genuine problem of quantum gravity, only the semiclassical theory has been considered. All scenarios put forward so far thus only rest on speculations. To address these issues properly, one should start from a specific approach to quantum gravity such as the Wheeler-DeWitt equation (2.1). While it is not yet clear how to apply this equation to the black hole case one can at least apply the corrected Schrödinger equation, see (3.11), and hope that the results obtained indicate some genuine features of quantum gravity.

As discussed above, the functional Schrödinger equation (2.38) describes the level of quantum field theory on a classical gravitational background, i. 
e. the level where the Hawking effect is calculated, see, e. g., Freese, Hill, and Mueller (1985) for a discussion in the functional Schrödinger picture. We choose a slicing of the background spacetime, which is obtained from $S_{0}$, such that $R=0$. How can the correction terms in (3.11) then be evaluated? An important observation is that one deals here with an asymptotically flat space, in contrast to the compact case of the general discussion. The WheelerDeWitt equation (2.1), if integrated over space, is thus not correct as it stands but has to be supplemented by a surface term from infinity. This is nothing but the ADM mass, $\mathcal{M}$. One thus has to replace the term $\sqrt{h} R$ in the denominators of (3.11) by $-16 \pi G \mathcal{M} / c^{2}$. This is already clear from dimensional arguments, since $\sqrt{h} R$ has dimensions of a length and the only length scale in the black hole case is the Schwarzschild radius.

One might wonder whether the functional Schrödinger equation (2.38) can be recovered at all in this case, since the flat slicing of Schwarzschild spacetime corresponds to a solution $S_{0}=$ constant of the Hamilton-Jacobi equation, and the whole spacetime would thus only be a point in the configuration space. This would certainly be the case for a compact space, but in the asymptotically flat case a distinguished time parameter is associated with the Poincaré group at infinity. This time parameter is the one that shows up in the Schrödinger equation (2.38). It is also the time parameter with respect to which the question of unitarity is discussed in the case of black hole evaporation.

The ratio of the first correction term in (3.11) to the dominant term, which is $\mathcal{H}_{m}$, is given by the ratio of the energy of the scalar field to $\mathcal{M} c^{2}$, which is small even if the mass of the hole approaches the Planck regime. This is not the case for the second term! It contributes the following term to the Hamiltonian:

$$
\Delta H_{m} \equiv-\frac{4 \pi i G \hbar}{c^{4}} \int d^{3} x \mathcal{H}_{m} \frac{\partial}{\partial t}\left(\frac{c^{2}}{16 \pi G \mathcal{M}(t)}\right) .
$$

We have here taken into account only the time derivative of the ADM mass, since it is the change of the background geometry which is the relevant contribution in the final stage of the evaporation (the decrease of the ADM mass due to Hawking evaporation). The time dependence of the mass of the black hole can be obtained by using the expression for the Hawking temperature and Stefan-Boltzmann's law, see e. g. DeWitt (1975). This leads to

$$
\mathcal{M}(t)=\left(\mathcal{M}_{0}^{3}-\frac{a \hbar c^{4}}{G^{2}} t\right)^{1 / 3},
$$

where $\mathcal{M}_{0}$ is the initial mass of the hole, and $a$ is a numerical factor that depends on the details of the model (the number of particle species considered, etc.) and whose precise value is not important for the present qualitative 
discussion. Eq. (3.37) can of course only be used if one assumes that the black hole does not settle to a mass which is much bigger than the Planck mass.

The term (3.37) becomes important if its of the same order of magnitude than the Hamiltonian $H_{m}$ of the scalar field, i. e. if

$$
\frac{\hbar}{4 M^{2} c^{2}} \frac{\partial \mathcal{M}}{\partial t} \approx\left(\frac{m_{P l}}{\mathcal{M}}\right)^{4} \approx 1
$$

The non-unitary term thus becomes important if the mass of the evaporating black hole approaches the Planck mass. In the above scenario, this happens after a time

$$
\bar{t} \approx\left(\frac{\mathcal{M}_{0}}{m_{P l}}\right)^{3} t_{P l} .
$$

After the mass of the hole has entered the Planck regime, the semiclassical expansion breaks down and one would have to deal with the full WheelerDeWitt equation. Nevertheless, the correction term (3.37) may be a good approximation if, for example, the black hole settles to a remnant of the order of the Planck mass but such that the numerical value of (3.37) is still small compared to $H_{m}$. It may also happen that the final evaporation time is of the order of $\mathcal{M}_{0}^{4}$ (Carlitz and Willey, 1987) which would mean that (3.37) is an excellent approximation over a long period of time.

A non-hermitean Hamiltonian does not transform a pure state into a mixed state. It does, however, change the "degree of purity" for a density matrix. In the present case its sign is such that it increases the degree of purity, if the mass of the hole decreases, see Kiefer, Müller, and Singh (1993). Whether this can be interpreted as a hint for the unitarity of quantum gravity is an open issue.

Although the above considerations are of a heuristic nature, they nevertheless demonstrate that the correction terms to the Schrödinger equation can be applied to concrete physical examples. 


\section{Chapter 4}

\section{Decoherence and back reaction}

In the derivation of the Schrödinger equation from quantum gravity, as it was presented in section 2, a crucial assumption was that the gravitational part of the wave functional is of the very special form $D^{-1} \exp \left(i M S_{0} / \hbar\right)$. The use of such a special, complex, solution to the real Wheeler-DeWitt equation (2.1) has been criticised in particular by Barbour (1993), who regarded this choice as being equivalent to putting in the desired result, namely the timedependent Schrödinger equation, by hand. The purpose of this section is to demonstrate that the use of such a single WKB state can be justified in a very natural, physical, way.

Consider, for example, a solution to (2.1) at order $M^{0}$ which is a superposition of the state (2.40) with its complex conjugate,

$$
\Psi=\frac{1}{D} \exp \left(i M S_{0} / \hbar\right) \chi\left[h_{a b}, \phi\right]+\frac{1}{D} \exp \left(-i M S_{0} / \hbar\right) \chi^{*}\left[h_{a b}, \phi\right] .
$$

Both $\chi$ and $\chi^{*}$ obey a time-dependent Schrödinger equation, where time is defined respectively by $S_{0}$ and $-S_{0}$. The superposition (4.1), however, does not describe a classical world, since there are interference terms between both components of (4.1). Note that the superposition (4.1) is analogous to the superposition (2.6) in the derivation of the Born-Oppenheimer approximation.

How can one get rid of these "unwanted" superpositions? The basis idea is the fact that states like (4.1) are highly correlated in the high-dimensional configuration space of gravitational and non-gravitational degrees of freedom, but that only very few of them are actually accessible to a localised observer. Instead of the full state (4.1) such an observer has thus to discuss the reduced density matrix, which is obtained from (4.1) by integrating out the (huge number of) irrelevant degrees of freedom. States of the accessible part, which are then correlated with orthogonal states of the "rest of the world" do not exhibit any interference terms locally. Information about the corresponding phase relations has been delocalised in the full configuration 
space. This mechanism is referred to as decoherence. In the derivation of the Born-Oppenheimer approximation (2.8), decoherence lies at the heart of the neglection of interference terms.

In the following I will first briefly discuss two examples from quantum mechanics and then show how decoherence can justify the use of only one of the components in (4.1). For a general discussion on decoherence I refer to the extensive literature on this subject - see, for example, Zeh $(1992,1993 \mathrm{a})$ or Zurek (1991), and the references therein.

\subsection{Decoherence in quantum mechanics: Two examples}

The first example is the localisation of macroscopic objects through interaction with their natural environment, for example through scattering by photons or air molecules (Joos and Zeh, 1985). Be $|\mathbf{x}\rangle$ the centre of mass position eigenstate of the macroscopic body, and $|\chi\rangle$ an initial state of the environment such that the total state at, say, $t=0$ is a product of these states. The interaction be such that this state evolves according to

$$
|\mathbf{x}\rangle|\chi\rangle \stackrel{t}{\rightarrow}|\mathbf{x}\rangle S_{\mathbf{x}}|\chi\rangle
$$

where $S_{\mathbf{x}}$ denotes the scattering matrix. The state of the environment is thus correlated with the state of the body. If one starts now from an entangled, nonclassical, state for the body, it will envolve, according to the superposition principle, into an entangled state of the body with the environment, i. e.

$$
\left(\int d^{3} x \varphi(\mathbf{x})|\mathbf{x}\rangle\right)|\chi\rangle \stackrel{t}{\rightarrow} \int d^{3} x \varphi(\mathbf{x})|\mathbf{x}\rangle S_{\mathbf{x}}|\chi\rangle .
$$

There is no sign of any localisation for the body in this state! Since, however, the huge number of degrees of freedom of the environment is inaccessible to a local observer, the relevant object is the density matrix of the position of the scattering centre after scattering:

$$
\begin{aligned}
\rho\left(\mathbf{x}, \mathbf{x}^{\prime}\right) & =\varphi(\mathbf{x}) \varphi^{*}\left(\mathbf{x}^{\prime}\right)\left\langle\chi\left|S_{\mathbf{x}^{\prime}}^{\dagger} S_{\mathbf{x}}\right| \chi\right\rangle \\
& \approx \varphi(\mathbf{x}) \varphi^{*}\left(\mathbf{x}^{\prime}\right) \exp \left(-\Lambda t\left(\mathbf{x}-\mathbf{x}^{\prime}\right)^{2}\right)
\end{aligned}
$$

The Gaussian factor in (4.4) leads to the suppression of interference effects between different positions of the scattered object ( $\Lambda$ contains the details of the scattering process) - the object becomes localised, if the mass of the body is sufficiently large, and wave packets are prevented from spreading. The density matrix in (4.4) obeys a non-unitary master equation instead of a unitary von Neumann equation. As shown by Joos and Zeh (1985), 
even the scattering by the photons of the microwave background leads to the localisation of a dust grain in interstellar space.

Two important aspects of decoherence should be stressed (Zeh, 1993b). The first aspect follows from the very formalism of decoherence and is independent of any interpretation. It states that the phase relations of an entangled state like (4.3) can never be seen by a local observer according to the probabilistic rules used in conventional quantum theory. This does not mean, however, that only one component of this state survives - all components are still "there." The second aspect addresses the issue of why only one component is "observed." This involves the interpretation of the quantum formalism. Basically, there are two options: Either one has to assume that the decohered components are still co-existing, but are dynamically independent from each other (this dynamical independence is what decoherence achieves) or one has to invoke explicit collapse models (and thereby has to change conventional quantum theory) to single out a specific component, which is then the "observed one." For all practical purposes, this is equivalent to the branching into dynamically independent components in the first interpretation, where decoherence defines an ensemble of wave functions, the "preferred basis" (Zurek, 1993).

The second example is taken from nuclear physics, where deformed nuclei exhibiting a definite orientation can be found even if the total quantum state is an angular momentum eigenstate (Zeh 1967, 1993b). Consider an $N$-nucleon system which is described by a stationary Schrödinger equation. An approximate solution, as it is found through an appropriate variation principle, is given by the antisymmetrised produced, $\phi$, of one-particle nucleon wave functions (Slater determinants). Since a superposition of different such $\phi$ is not a determinant again (due to the nonlinearity of the ansatz), these solutions need not be eigenstates of an operator like angular momentum which commutes with the Hamiltonian. They may thus describe a deformed nucleus, which has a definite orientation. Performing a superposition of different "deformed solutions,"

$$
\Psi=\int d \Omega f(\Omega) U(\Omega) \phi
$$

where $U(\Omega)$ denotes a unitary representation of the rotation group and $\Omega$ may stand, e. g., for the Euler angles, one obtains, of course, again an approximate solution to the stationary Schrödinger equation. The amplitude $f(\Omega)$ may be chosen such that $\Psi$ is an approximate eigenstate of angular momentum.

If many nucleons are present in the nucleus, a certain nucleon is correlated with a corresponding deformed potential generated by the remaining nucleons in each component of the superposition (4.5) ("strong coupling"). The matrix 
element between different orientations reads"

$$
\begin{aligned}
\left\langle U(\Omega) \phi \mid U\left(\Omega^{\prime}\right) \phi\right\rangle & =\prod_{i=1}^{N}\left\langle U(\Omega) \phi_{i} \mid U\left(\Omega^{\prime}\right) \phi_{i}\right\rangle \\
& \propto \exp \left(-\lambda\left(\Omega-\Omega^{\prime}\right)^{2}\right) .
\end{aligned}
$$

For large $N$ this is a narrow Gaussian peaked around $\Omega=\Omega^{\prime}$ - the different orientations in (4.5) have decohered from one another. This is an example of a spontaneous symmetry breaking. As can be seen from the existence of a rotational spectrum, these different orientations even seem to rotate slowly, in spite of the stationary nature of the total state! This is the analogue to the recovery of an approximate time-dependent Schrödinger equation for intrinsic degrees of freedom from the stationary Wheeler-DeWitt equation (2.1). Since $\left\langle U\left(\Omega^{\prime}\right) \phi \mid U(\Omega) \phi\right\rangle \approx \delta\left(\Omega-\Omega^{\prime}\right)$, one has $\langle\phi(\Omega) \mid \psi\rangle \approx f(\Omega)$ and can thus assign an approximate wave function $f(\Omega)$ to the rotational degrees of freedom, describing a fictitious "rigid top."

If the nucleus contained an intrinsic quantum observer, such an observer would see the nucleus with a definite orientation. In the case of quantum cosmology, where the Wheeler-DeWitt equation (2.1) is applied to the whole Universe, all observers are intrinsic observers, and decoherence is the reason why they observe a classical world. This is discussed in the next subsection.

\subsection{Decoherence of different WKB branches in quantum gravity}

The analogue of the rotational symmetry of the nuclear Hamiltonian is here the (discrete) symmetry of the Wheeler-DeWitt Hamiltonian in (2.1) under complex conjugation. The semiclassical expansion presented in the previous sections shows that, to order $M^{0}$, the functionals $\chi\left[h_{a b}, \phi\right]$ of the nongravitational fields obey an approximate time-dependent Schrödinger equation, which is a complex equation! Consequently, $\chi$ is in general complex and $\chi$ and $\chi^{*}$ thus couple drastically different to the components $\exp \left(i M S_{0} / \hbar\right)$ and $\exp \left(-i M S_{0} / \hbar\right)$. Since a huge number of degrees of freedom is involved, this leads to decoherence between the two components in (4.1), if these degrees of freedom are integrated out to get the reduced density matrix

$$
\rho\left[h_{a b}, h_{a b}^{\prime}\right] \equiv \operatorname{Tr}_{\phi} \Psi\left[h_{a b}, \phi\right] \Psi^{*}\left[h_{a b}^{\prime}, \phi\right]
$$

for the gravitational field. This general expectation is verified in concrete models (see Kiefer (1992c) for a review). Typically, global degrees of freedom

\footnotetext{
${ }^{1}$ We assume here for simplicity that $\phi=\prod \phi_{i}$ (no antisymmetrisation).

${ }^{2}$ Other important examples are chiral molecules and the Higgs vacuum, see Zeh (1992).
} 
like the scale factor $a$ of a Friedmann Universe are chosen to represent the semiclassical gravitational degrees of freedom, while "small fluctuations" like density perturbations or gravitational waves play the role of the irrelevant degrees of freedom, which are integrated out. The functional $\chi$ is written in these examples as

$$
\chi=\prod_{n=1}^{N} \chi_{n}\left(a ; x_{n}\right),
$$

where the $x_{n}$ may be, for example, coefficients in the expansion of the scalar field $\phi$ into its harmonics. The result is that $\rho$ becomes a narrow Gaussian in $a-a^{\prime}$ (this is the analogue of the quantum mechanical example on localisation and may here be described as a "localisation within one WKB component" or "measurement of the three-geometry") and contains in addition a large suppression factor for the interference term between the WKB components in (4.1) (this is the analogue of the deformed nucleus or the chiral molecule). There are, however, situations where $\chi$ is approximately real and the two components in (4.1) interfere. This happens, for example, in regions which would correspond in the classical theory to the turning point of a recollapsing universe (Kiefer, 1992b), where $\partial S_{0} / \partial a$ becomes small. Decoherence thus justifies the selection of a definite WKB component in the semiclassical approximation as well as the localisation within one component. This is, however, not yet sufficient to justify the notion of a classical spacetime. It requires in addition the persistence of decoherence along a whole WKB trajectory and is related to the issue of back reaction which will be discussed in the next subsection.

I want to remark finally that it is of course not necessary to deal only with the whole Universe in quantum gravity. A quantum black hole, for example, may be viewed from outside and is thus a more direct analogue of the deformed nucleus.

\subsection{Back reaction}

In this section I want to address the question to what extent the presence of the non-gravitational matter fields lead to a modification of the HamiltonJacobi equation (2.29) at order $M^{-1}$, i. e. the question of back reaction. In particular, I want to discuss the conditions under which this back reaction is given by the expectation value of the Hamiltonian (2.25) with respect to the state $\chi$ satisfying the approximate Schrödinger equation. This is equivalent to study the range of validity of the semiclassical Einstein equations

$$
G_{\mu \nu}=\frac{8 \pi G}{c^{4}}\left\langle T_{\mu \nu}\right\rangle
$$

At order $M$, the value of the geometrodynamical momentum when applied to the WKB state of the gravitational field is given by the familiar expression 
(2.30). To find out how this expression may be changed at the next order, we first decompose $\chi$ into its absolute value and its phase,

$$
\chi \equiv R \exp (i \theta / \hbar)
$$

and then rewrite the Hamilton-Jacobi equation (2.29) as follows:

$$
\frac{1}{2 M} G_{a b}\left(M \frac{\delta S_{0}}{\delta h_{a}}+\frac{\delta \theta}{\delta h_{a}}\right)\left(M \frac{\delta S_{0}}{\delta h_{b}}+\frac{\delta \theta}{\delta h_{b}}\right)+M V-\frac{\delta \theta}{\delta \tau}+\mathcal{O}\left(M^{-1}\right)=0 .
$$

Performing the expectation value of this equation with respect to $\chi$ and introducing the quantity

$$
\Pi^{a} \equiv M \frac{\delta S_{0}}{\delta h_{a}}+\left\langle\chi \mid \frac{\delta \theta}{\delta h_{a}} \chi\right\rangle
$$

this can be written as

$$
\frac{1}{2 M} G_{a b} \Pi^{a} \Pi^{b}+M V-\left\langle\chi \mid \frac{\delta \theta}{\delta \tau} \chi\right\rangle+\mathcal{O}\left(M^{-1}\right)=0 .
$$

The crucial assumption made at this stage is that $\Pi^{a}$ is the geometrodynamical momentum at this order of approximation. We will specify later the conditions under which this assumption is valid. Taking this for granted, Eq. (4.13) is the desired "back reaction corrected" Hamilton-Jacobi equation. It can be conveniently rewritten by noting that

$$
\left\langle\chi \mid \frac{\delta \theta}{\delta \tau} \chi\right\rangle=-\left\langle\chi \mid \mathcal{H}_{m} \chi\right\rangle
$$

which follows immediately from the fact that $\theta$ is the phase of a solution to the time-dependent Schrödinger equation (2.38). One can thus write (4.13) as

$$
\frac{1}{2 M} G_{a b} \Pi^{a} \Pi^{b}+M V+\left\langle\chi \mid \mathcal{H}_{m} \chi\right\rangle+\mathcal{O}\left(M^{-1}\right)=0
$$

Making use of (2.30) and (2.38) one can write

$$
\left\langle\chi \mid \mathcal{H}_{m} \chi\right\rangle=-\frac{\hbar}{M} G_{a b} \Pi^{a} A^{b}
$$

where we have introduced in analogy to (2.9) a "super Berry connection" (see Balbinot, Barletta, and Venturi, 1990; Datta, 1993; Kiefer, 1993a)

$$
A^{b}=-i\left\langle\chi \mid \frac{\delta \chi}{\delta h_{b}}\right\rangle
$$

which is defined on the space of three-metrics. 
An equivalent way to write (4.13) is then, up to terms of the order $M^{-1}$,

$$
\begin{aligned}
& \left\{\frac{1}{2 M} G_{a b}\left(-i \hbar \frac{\delta}{\delta h_{a}}-\hbar A^{a}\right)\left(-i \hbar \frac{\delta}{\delta h_{b}}-\hbar A^{b}\right)+M V\right. \\
& \left.-\frac{\hbar^{2}}{2 M} G_{a b} A^{a} A^{b}+\mathcal{O}\left(M^{-1}\right)\right\} \psi_{G}=0
\end{aligned}
$$

where $\psi_{G}$ is an effective gravitational wave function at this order of approximation. I emphasize that this equation is very similar to the equation (2.8) for the nuclear wave function in the Born-Oppenheimer approximation. The term quadratic in the connection, which is already of order $M^{-1}$, has only been retained in (4.18) because of this comparison.

An important consequence of the back reaction is that the notion of WKB time is modified since an additional term proportional to $M^{-1}$ arises from (4.12). This term is thus of the same order as the corrections (3.11) to the Schrödinger equation. Using the definition (4.12), one can rewrite the time derivative introduced in (2.38) as follows:

$$
\begin{aligned}
i \hbar \frac{\delta \psi}{\delta \tau} & \equiv i \hbar G_{a b} \frac{\delta S_{0}}{\delta h_{a}} \frac{\delta \psi}{\delta h_{b}}=i \hbar G_{a b}\left(\frac{\Pi^{a}}{M}-\frac{1}{M}\left\langle\chi \mid \frac{\delta \theta}{\delta h_{a}} \chi\right\rangle\right) \frac{\delta \psi}{\delta h_{b}} \\
& \equiv i \hbar \frac{\delta \psi}{\delta \tilde{\tau}}-\frac{i \hbar}{M} G_{a b}\left\langle\chi \mid \frac{\delta \theta}{\delta h_{a}} \chi\right\rangle \frac{\delta \psi}{\delta h_{b}} .
\end{aligned}
$$

At order $M^{-1}$ the "back reaction corrected" WKB time $\tilde{\tau}$ has to be used instead of the old $\tau$. To find the induced contribution of this change in the definition of time to the corrected Schrödinger equation (3.11), we first decompose the vector field $G_{a b} \delta \psi / \delta h_{a}$ as in (3.9) into its components tangential and orthogonal to the flow lines of the vector field $G_{a b} \delta S_{0} / \delta h_{a}$ and assume, again, that the orthogonal component is much smaller than the tangential component. Thus,

$$
G_{a b} \frac{\delta \psi}{\delta h_{a}}=\frac{i}{2 \hbar V} \mathcal{H}_{m} \psi G_{a b} \frac{\delta S_{0}}{\delta h_{a}}+\mathcal{O}\left(M^{-1}\right) .
$$

With the help of (4.14) one can then write (4.19) in the form

$$
i \hbar \frac{\delta \psi}{\delta \tau}=i \hbar \frac{\delta \psi}{\delta \tilde{\tau}}-\frac{1}{2 M V}\left\langle\chi \mid \mathcal{H}_{m} \chi\right\rangle \mathcal{H}_{m} \psi
$$

so that the corrected Schrödinger equation (3.11) can be written as an equation with respect to the new time $\tilde{\tau}$ :

$$
\begin{aligned}
i \hbar \frac{\delta \psi}{\delta \tilde{\tau}}= & \mathcal{H}_{m} \psi+\frac{4 \pi G}{c^{4} \sqrt{h}(R-2 \Lambda)}\left(\mathcal{H}_{m}^{2}-2\left\langle\mathcal{H}_{m}\right\rangle \mathcal{H}_{m}\right) \psi \\
& +i \hbar \frac{4 \pi G}{c^{4}} \frac{\delta}{\delta \tau}\left(\frac{\mathcal{H}_{m}}{\sqrt{h}(R-2 \Lambda)}\right) \psi
\end{aligned}
$$


The modification in the definition of time thus leads to an additional contribution to the shift of energies induced by quantum gravity. In our example of the scalar field in de Sitter space (section 3.2.2) this additional term leads to a change of sign in the shift of the expectation value for the Hamiltonian density. Instead of (3.34) one has

$$
\left\langle\mathcal{H}_{m}\right\rangle \rightarrow\left\langle\mathcal{H}_{m}\right\rangle+\frac{841}{1382400 \pi^{3}} \frac{G \hbar H_{0}^{6} a^{3}}{c^{8}}
$$

From the corrected Hamilton-Jacobi equation (4.15) one finds in this example, using (3.30), an effective increase of the Hubble parameter

$$
H_{0}^{2} \rightarrow H_{0}^{2}\left(1+\frac{29}{360 \pi} \frac{G H_{0}^{2} \hbar}{c^{5}}\right)
$$

The increase in $H_{0}$ is thus essentially determined by the square of the ratio of the Planck length to the horizon length. If our present Universe were in a de Sitter phase this increase in $H_{0}$ would be of the order of $10^{-120}$.

Analogus to the Hamilton-Jacobi equation one gets a correction to the momentum constraints at this order of approximation, i. e.

$$
D_{a} \Pi^{a b}=\left\langle\chi \mid T_{0}^{b} \chi\right\rangle
$$

One has thus recovered the semiclassical Einstein equations (4.9) at order $M^{-1}$.

What are the exact conditions under which the semiclassical HamiltonJacobi equation is valid? The first, obvious, condition is that the expectation value of $\mathcal{H}$ with respect to the state satisfying the approximate Schrödinger equation be small compared to the other terms in (4.15). This came out in a natural way through the expansion scheme with respect to the Planck mass. The second condition, as has been discussed in section 4.1, is the smallness of interference terms between different WKB components of the total state. This is the analogy of the smallness of interferences between the different components of (2.6) and is a necessary condition for the validity of a Born-Oppenheimer approximation. As shown in section 4.1, these various components decohere in realistic cases and thereby justify the use of a single WKB component.

The third, and most subtle, condition is the possibility to replace the geometrodynamical momentum at order $M^{-1}$ by the expression (4.12). This can be done if there is a strong correlation in the gravitational sector between the momentum and the expression on the right-hand side of (4.12). There is no simple general condition for this to be the case. The usual approach is to employ the Wigner function for the reduced density matrix (4.7) obtained by integrating out non-gravitational degrees of freedom within a single WKB 
component. For a given density matrix in the position representation $\rho\left(q, q^{\prime}\right)$, the Wigner function is defined as

$$
F_{W}(\bar{q}, p)=\int d \Delta e^{-2 i p \Delta} \rho(\bar{q}+\Delta, \bar{q}-\Delta)
$$

where $\bar{q}=\left(q+q^{\prime}\right) / 2$ and $\Delta=\left(q-q^{\prime}\right) / 2$. The Wigner function is the closest one can get to a classical phase space distribution; because of the quantum uncertainty, the Wigner function is in general not positive definite. The Wigner function has been used in quantum gravity by several authors including Halliwell (1987), Padmanabhan and Singh (1990), Habib and Laflamme (1990), and Paz and Sinha (1991). To evaluate (4.26) one has to first calculate the reduced density matrix by using a definite solution of the Schrödinger equation satisfied by $\chi$. In most examples discussed so far a Gaussian solution such as (3.23) has been chosen. The Wigner function (4.26) can then be evaluated and is in these examples typically of the form

$$
\begin{aligned}
F_{W}\left[h_{a}, \Pi^{a}\right] \approx & D^{-2} \sqrt{\frac{\pi}{\sigma^{2}}} \exp \left[-\frac{1}{\sigma^{2}} G_{a b}\left(\Pi^{a}-M \frac{\delta S_{0}}{\delta h_{a}}-\left\langle\chi \mid \frac{\delta \theta}{\delta h_{a}} \chi\right\rangle\right)\right. \\
& \left.\left(\Pi^{b}-M \frac{\delta S_{0}}{\delta h_{b}}-\left\langle\chi \mid \frac{\delta \theta}{\delta h_{b}} \chi\right\rangle\right)\right],
\end{aligned}
$$

where

$$
\sigma^{2}=\operatorname{Tr} \frac{|\delta \Omega / \delta \tau|^{2}}{4 \operatorname{Re} \Omega^{2}}
$$

turns out to be the inverse of the coherence width associated with the reduced density matrix itself. ( $\Omega$ is the covariance of the Gaussian, see (3.23).) The conditions for the validity of the semiclassical Hamilton-Jacobi equation arising from these considerations are thus a) that the width (4.28) of the Gaussian (4.27) is much smaller than the peak value of the Gaussian ("strong correlation condition") and b) that the inverse width is much smaller than the components of the three-metric which are "measured" by the huge number of irrelevant degrees of freedom ("strong decoherence condition"). Typically, these conditions are fulfilled if the fluctuation of the Hamiltonian density is small in the state $\chi$ satisfying the approximate Schrödinger equation, i. e. if (3.33) holds. Under this condition, therefore, one would expect the expressions derived within this framework to hold very accurately. It is, however, still unclear what are the most general conditions which are compatible with the back reaction-corrected Hamilton-Jacobi equation (4.15). 


\section{Chapter 5}

\section{Discussion}

It is my purpose in this contribution to present, as I hope convincing, arguments that it is possible to extract physical insight from semiclassical gravity even if the underlying exact theory is not yet available. The formal framework is based on the canonical approach to quantum gravity and its central equation (2.1). Although the fundamental Hamiltonian may not be exactly of the form (2.1), general principles like the reparametrisation invariance of the classical theory suggest that a constraint equation of the form $H \Psi=0$ will still play a central role. Semiclassical considerations may thus well be, at least to a large extent, independent of the concrete form of the fundamental equation. Moreover, the necessary requirement that the, well-tested, Schrödinger equation can be recovered as an approximate equation puts an important restriction on the fundamental theory. Corrections to the Schrödinger equation, on the other side, may turn out to be different from one theory to the other and thus allow the possibility to distinguish between alternative approaches. I emphasise that such correction terms lead to definite physical predictions such as the energy shift in (4.23) which can, at least in principle, be checked and thus confirmed or falsified.

I want to finally give a brief outlook on other important topics in semiclassical gravity which have not been addressed in this contribution. The first topic has to do with the relevance of semiclassical considerations for the problem of time in quantum gravity (Isham, 1992; Kuchař, 1992). Briefly speaking, the problem of time in quantum gravity is concerned with the "timeless nature" of the Wheeler-DeWitt equation (2.1) (by which one means the absence of the classical time parameter $t$ ) and the question whether a physical concept of time can be introduced at the most fundamental level or only in the semiclassical regime. I have presented an approximation scheme where the Schrödinger equation together with its classical time parameter emerges in an appropriate limit. The expansion scheme is only valid if the higher order terms are small compared to the dominant one. The semiclassical Einstein equation (4.9), in particular, is only valid if the right-hand side is treated as a 
small perturbation. A nonperturbative treatment would in general not lead to sensible results. This argument also applies to the corrected Schrödinger equation (3.11). One would thus not expect that this semiclassical notion of time can be extrapolated to the full theory. On the contrary, the fact that the Schrödinger equation and with it all the concepts of ordinary quantum theory like Hilbert space and unitarity are only recovered approximately (recall (3.14)) leads me to the suspicion that these concepts do not play any fundamental role in quantum gravity. As mentioned before, the presence of a Hilbert space structure on the level of the exact theory may be in conflict with results from semiclassical expansions, see Louko (1993). Interpretations of quantum gravity which only work in the semiclassical regime, like the one by Vilenkin (1989), do in my opinion not really solve the problem of time and may even present an obstacle on the way to finding the correct full theory.

There is one attempt to extrapolate the semiclassical concept of time to full quantum gravity which has gained some attention, see Padmanabhan (1990), Greensite (1990), and Squires (1991). The basic idea there is to extract time from the phase of the full wave functional. In the semiclassical regime it agrees, of course, with the WKB time in the approximate Schrödinger equation, but it can be drastically different in other cases. This "phase time" parametrises, at least locally, a flow in the configuration space. This flow does not, in general, have anything to do with the background spacetime which was reconstructed from a given solution to the HamiltonJacobi equation (2.29). The main advantage of this approach seems to be that an exact Ehrenfest equation can be written down for the expectation value of an operator depending on the variables which are comoving with respect to the "phase time flow." The expectation value is performed with respect to the Schrödinger inner product of the full wave functional and the comoving variables. There are, however, some problems with this approach. Firstly, the procedure does not work for real wave functionals which can therefore not be interpreted in this framework. Secondly, the concept of phase time depends on the given solution for the Wheeler-DeWitt equation and may thus seem inappropriate as a fundamental concept. Thirdly, phase time does not exist globally and may also lead to the "spacetime problem," i. e. the dependence of its definition upon the choice of the original spacetime foliation in the canonical approach (Kuchař, 1992). I emphasise again that this problem did not occur in the semiclassical approximation. Finally, the Ehrenfest condition with respect to the momentum variables does only hold if the phase of the wave functional varies rapidly along the flow lines which it defines. In the special case, however, where the semiclassical expansion scheme converges, it seems that the sum over all orders leads to a modification in the original WKB time which just yields the above phase time. This is spelled out elsewhere (Kiefer, 1993c).

Can anything be said about the concept of time on the fundamental level 
of (2.1) itself? The answer is yes, since the structure of the DeWitt metric in front of the kinetic term distinguishes a certain variable in configuration space from the remaining ones by its minus sign. At each space point, (2.1) is thus of the hyperbolic type. But only in the case where one global minus sign survives after the diffeomorphism group has been factored out could one say that (2.1) itself is hyperbolic. This is, however, not the case in the full configuration space, but only in special domains (Giulini, 1993). One important example is the neighbourhood of Friedmann-type three-geometries for constant lapse. In this case there exists a global intrinsic timelike variable, the scale factor of the Friedmann space, with respect to which a well-defined initial value problem can be posed for the wave functional (Zeh 1988, 1992a). There is, however, no unitary evolution with respect to this intrinsic time. This fact leads to an interesting tension between intrinsic time and WKB time which has drastic consequences in the case of a recollapsing Friedmann Universe (Zeh, 1992; Kiefer, 1993d): The semiclassical approximation can not be valid along the whole region of the corresponding classical trajectory and genuine quantum effects of gravity become important far away from the Planck scale.

I want to finally make a brief comparison of the semiclassical approximation to quantum gravity in the canonical approach with the covariant effective action approach ("background field method"). Formally, the level of the approximate functional Schrödinger equation corresponds to the level of the one-loop effective action. Taking into account the correction terms of (3.11) is analogous to the two-loop level of the effective action, although this has not yet been explicitly demonstrated. There is, however, a conceptual difference. The effective action approach starts from a given background spacetime which in the canonical framework was only recovered under appropriate circumstances such as the decoherence between different WKB components. The background field method therefore can be applied only under these circumstances.

\section{Acknowledgement}

I thank the organisers for inviting me to this workshop and the participants

for many interesting discussions. I have attempted to address their questions and comments in my contribution to these Proceedings.

\section{References}

A. Ashtekar (1991): Lectures on non-perturbative canonical gravity (World Scientific, Singapore). 
A. Ashtekar (1988): New perspectives in canonical gravity (Bibliopolis, Napoli).

R. Balbinot, A. Barletta, and G. Venturi (1990): Matter, quantum gravity, and adiabatic phase. Phys. Rev. D 41, 1848.

T. Banks (1985): TCP, Quantum Gravity, the Cosmological Constant, and all that .... Nucl. Phys. B249, 332.

J. B. Barbour (1992): Private communication.

J. B. Barbour (1993): Time and complex numbers in canonical quantum gravity. Phys. Rev. D 47, 5422.

E. P. Belasco and H. C. Ohanian (1969): Initial Conditions in General Relativity: Lapse and Shift Formulation. Journ. Math. Phys. 10, 1503.

O. Bertolami (1991): Nonlinear corrections to quantum mechanics from quantum gravity. Phys. Lett. A154, 225.

R. Brout and G. Venturi (1989): Time in semiclassical gravity. Phys. Rev. D 39, 2436.

R. D. Carlitz and R. D. Willey (1987): Lifetime of a black hole. Phys. Rev. D 36, 2336.

D. Cosandey (1993): Localized quantum mechanics in a semiclassical universe. University of Bern preprint.

T. Damour and J. H. Taylor (1991): On the orbital period change of the binary pulsar PSR 1913+16. Astrophys. Journal 366, 501.

D. P. Datta (1993): Semiclassical backreaction and Berry's phase. Mod. Phys. Lett. A 8, 191 (1993).

B. S. DeWitt (1967): Quantum Theory of Gravity I. The Canonical Theory. Phys. Rev. 160, 1113.

B. S. DeWitt (1970): "Spacetime as a sheaf of geodesics in superspace", in Relativity, ed. by M. Carmeli, S. Fickler, and L. Witten (Plenum, New York).

B. S. DeWitt (1975): Quantum field theory in curved spacetime. Phys. Rep. 19, 295.

K. Freese, C. T. Hill, and M. Mueller (1985): Covariant functional Schrdinger formalism and application to the Hawking effect. Nucl. Phys. B255, 693. 
U. H. Gerlach (1969): Derivation of the Ten Einstein Field Equations from the Semiclassical Approximation to Quantum Geometrodynamics. Phys. Rev. 177, 1929.

D. Giulini (1993): "What is the geometry of superspace?", in from Newton's bucket to quantum gravity, ed. by J. B. Barbour and H. Pfister (Birkhäuser, Boston).

J. Greensite (1990): Time and Probability in Quantum Cosmology. Nucl. Phys. B342, 409.

J. Guven, B. Lieberman, and C. T. Hill (1989): Schrödinger-picture field theory in Robertson-Walker flat spacetimes. Phys. Rev. D 39, 438.

S. Habib and R. Laflamme (1990): Wigner function and decoherence in quantum cosmology. Phys. Rev. D 42, 4056.

J. J. Halliwell (1987): Correlations in the wave function of the Universe. Phys. Rev. D 36, 3626.

J. J. Halliwell and S. W. Hawking (1985): Origin of structure in the Universe. Phys. Rev. D 31, 1777.

J. B. Hartle (1986): "Prediction in Quantum Cosmology", in Gravitation in Astrophysics, ed. by B. Carter and J. B. Hartle (Plenum Press, New York).

S. W. Hawking (1976): Breakdown of predictability in gravitational collapse. Phys. Rev. D 14, 2460.

W. Heisenberg and H. Euler (1936): Folgerungen aus der Diracschen Theorie des Positrons. Z. Phys. 98, 714.

C. J. Isham (1992): "Canonical Quantum Gravity and the Problem of Time", in Integrable Systems, Quantum Groups, and Quantum Field Theories (Kluwer Academic Publishers, London).

R. Jackiw (1988a): Berry's phase - Topological Ideas from Atomic, Molecular and Optical Physics. Comments At. Mol. Phys. 21, 71.

R. Jackiw (1988b): "Analysis on infinite-dimensional manifolds - Schrödinger representation for quantized fields", Field Theory and Particle Physics, ed. by O. Eboli, M. Gomes, and A. Santano (World Scientific, Singapore).

E. Joos and H. D. Zeh (1985): The Emergence of Classical Properties through Interaction with the Environment. Z. Phys. B59, 223. 
C. Kiefer (1987): Continuous measurement of mini-superspace variables by higher multipoles. Class. Quantum Grav. 4, 1369.

C. Kiefer (1992a): Functional Schrödinger equation for scalar QED. Phys. Rev. D 45, 2044.

C. Kiefer (1992b): Decoherence in quantum electrodynamics and quantum gravity. Phys. Rev. D 46, 1658.

C. Kiefer (1992c): "Decoherence in quantum cosmology", in Proceedings of the 10th Seminar on Relativistic Astrophysics and Gravitation, ed. by S. Gottlöber, J. P. Mücket, and V. Müller (World Scientific, Singapore).

C. Kiefer (1993a): Topology, decoherence, and semiclassical gravity. Phys. Rev. D 47, 5414.

C. Kiefer (1993b): "Semiclassical gravity with non-minimally coupled fields", in preparation.

C. Kiefer (1993c): "Semiclassical gravity and the problem of time", to appear in the Proceedings of the Cornelius Lanczos International Centenary Conference.

C. Kiefer (1993d): "Quantum cosmology and the emergence of a classical world", in Mathematics, Philosophy and Modern Physics, ed. by E. Rudolph and I.-O. Stamatescu (Springer, Berlin).

C. Kiefer and T. P. Singh (1991): Quantum Gravitational Corrections to the Functional Schrödinger Equation. Phys. Rev. D 44, 1067.

C. Kiefer, T. Padmanabhan, and T. P. Singh (1991): A comparison between semiclassical gravity and semiclassical electrodynamics. Class. Quantum Grav. 8, L 185.

C. Kiefer, R. Müller, and T. P. Singh (1993): Quantum Gravity and Non-unitarity in Black Hole Evaporation. Preprint gr-qc/9308024.

J. Kowalski-Glikman and J. C. Vink (1990): Gravity-matter mini-superspace: quantum regime, classical regime and in between. Class. Quantum Grav. 7, 901.

K. V. Kuchař (1970): Ground State Functional of the Linearized Gravitational Field. Journ. Math. Phys. 11, 3322.

K. V. Kuchař (1992): "Time and Interpretations of Quantum Gravity", In Proceedings of the 4th Canadian Conference on General Relativity and Relativistic Astrophysics, ed. by G. Kunstatter, D. Vincent and J. Williams (World Scientific, Singapore, 1992). 
C. Kuo and L. H. Ford (1993): Semiclassical gravity theory and quantum fluctuations. Phys. Rev. D 47, 4510.

C. Lämmerzahl (1993): Private communication.

L. D. Landau and E. M. Lifshitz (1975): Quantenmechanik (AkademieVerlag, Berlin).

V. G. Lapchinsky and V. A. Rubakov (1979): Canonical quantization of gravity and quantum field theory in curved space-time. Acta Physica Polonica B10, 1041.

J. Louko (1993): Holomorphic quantum mechanics with a quadratic Hamiltonian constraint. Phys. Rev. D 48, 2708.

V. Moncrief and C. Teitelboim (1972): Momentum Constraints as Integrability Conditions for the Hamiltonian Constraint in General Relativity. Phys. Rev. D 6, 966.

D. N. Page (1993): "Black Hole Information", to appear in the Proceedings of the 5th Canadian Conference on General Relativity and Relativistic Astrophysics, Waterloo, Ontario, May 1993.

T. Padmanabhan (1990): A Definition for Time in Quantum Cosmology. Pramana 35, L199.

T. Padmanabhan and T. P. Singh : On the semiclassical limit of the Wheeler-DeWitt equation. Class. Quantum Grav. 7, 411 (1990).

J. P. Paz and S. Sinha (1991): Decoherence and back reaction: The origin of the semiclassical Einstein equations. Phys. Rev. D 44, 1038.

A. Peres (1962): On Cauchy's Problem in General Relativity. Nuovo Cimento XXVI, 53.

E. Schrödinger (1926a): Quantisierung als Eigenwertproblem (Erste Mitteilung). Annalen der Physik 79, 361.

E. Schrödinger (1926b): Quantisierung als Eigenwertproblem (Vierte Mitteilung). Annalen der Physik 81, 109.

T. P. Singh (1990): Gravity induced corrections to quantum mechanical wave functions. Class. Quantum Grav. 7, L149.

T. P. Singh (1993): "Semiclassical gravity", in Advances in Gravitation and Cosmology, ed. by B. R. Iyer, A. R. Prasanna, R. K. Varma, and C. V. Vishveshwara (Wiley, Eastern, New Delhi). 
E. J. Squires (1991): The dynamical role of time in quantum cosmology. Phys. Lett. A155, 357.

A. Vilenkin (1989): Interpretation of the wave function of the Universe. Phys. Rev. D 39, 1116.

J. Wudka (1990): Remarks on the Born-Oppenheimer approximation. Phys. Rev. D 41, 712 (1990).

H. D. Zeh (1967): Symmetrieverletzende Modellzustände und kollektive Bewegungen. Z. Phys. 202, 38.

H. D. Zeh (1986): Emergence of Classical Time from a Universal Wave Function. Phys. Lett. A116, 9.

H. D. Zeh (1988): Time in Quantum Gravity. Phys. Lett. A126, 311.

H. D. Zeh (1992): The Physical Basis of The Direction of Time (Springer, Berlin).

H. D. Zeh (1993a): There are no quantum jumps, nor are there particles! Phys. Lett. A172, 189.

H. D. Zeh (1993b): "Decoherence and measurements", to appear in the Proceedings of Stochastic evolution of quantum states in open systems and measurement processes, Budapest, March 1993.

W. H. Zurek (1991): Decoherence and the Transition from Quantum to Classical. Phys. Today 44, 36.

W. H. Zurek (1993): Preferred States, Predictability, Classicality and the Environment-Induced Decoherence. Progr. Theor. Phys. 89, 281. 